\title{
Opportunities with fixed-target Drell-Yan
}

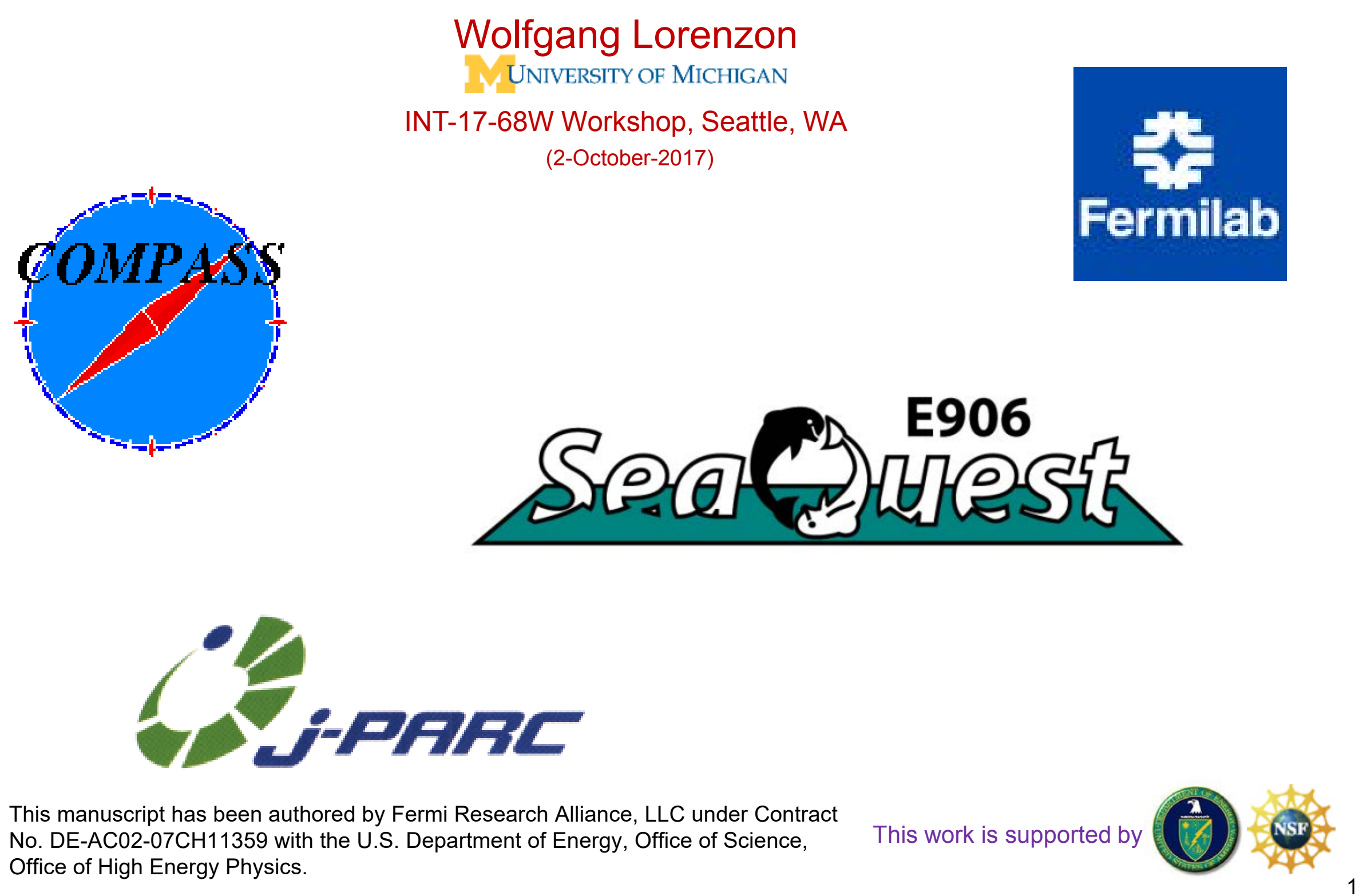




\section{Complementarity between SIDIS and Drell Yan}

- SIDIS and Drell-Yan have similar physics reach:

$\Rightarrow$ tools to probe quark and antiquark structure of nucleon

$\Rightarrow$ electromagnetic probes

SIDIS (spacelike)

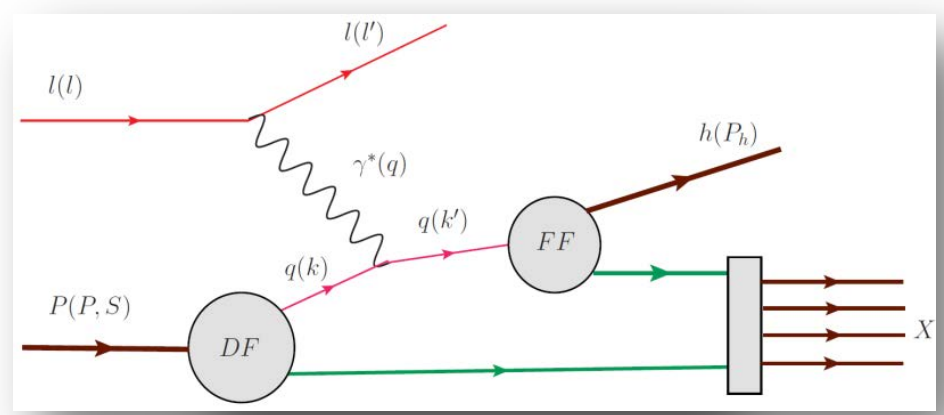

Quintessential probe of hadron structure:

$\rightarrow$ relatively simple to measure and calculate

$\Rightarrow$ charge-weighted flavor sensitivity

$\Rightarrow$ QCD final state effects

$\Rightarrow$ fragmentation process

$\Rightarrow$ no quark-antiquark selectivity
Drell-Yan (timelike) virtual photon

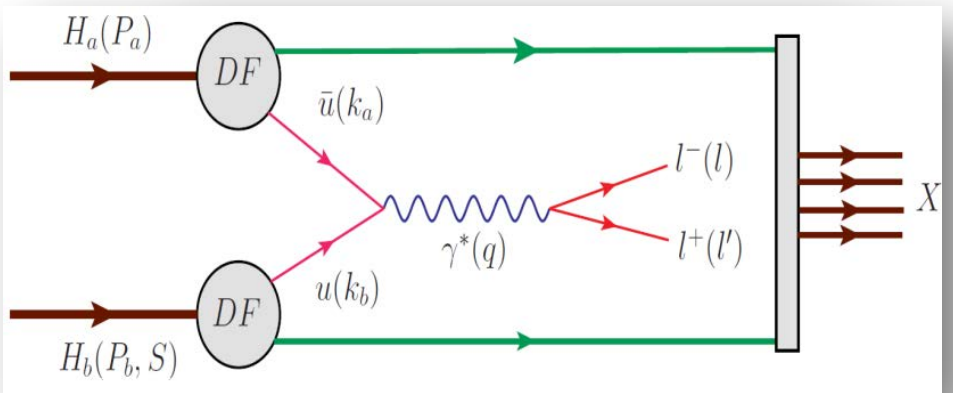

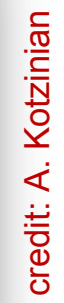

Cleanest probe to study hadron structure:

$\Rightarrow$ no QCD final state effects

$\Rightarrow$ no fragmentation process

$\Rightarrow$ production of two TMD parton distribution functions

$\rightarrow$ ability to select sea quark distribution

$\Rightarrow$ hadron beam: $\sigma(D Y) / \sigma($ nuclear $) \approx 10^{-7}$ 


\section{Factorization and Universality (SIDIS - DY)}

\author{
SIDIS \\ $\mathrm{PDF} \otimes \mathrm{FF}$
}

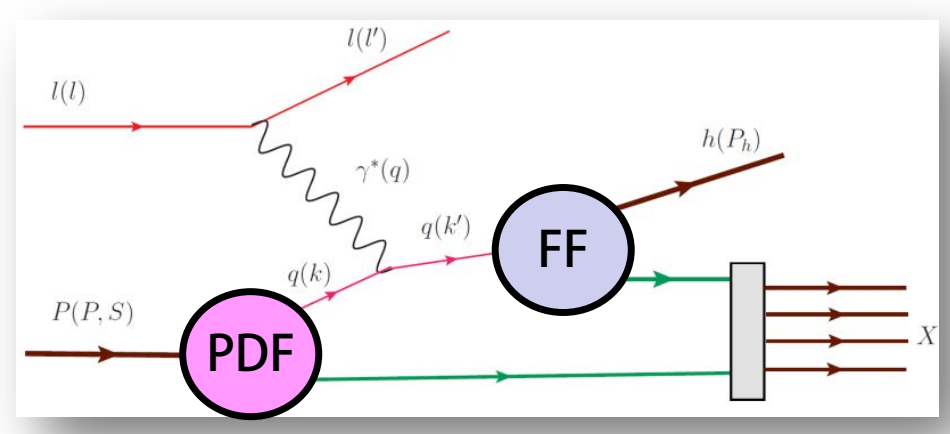

\author{
DY \\ $\mathrm{PDF} \otimes \mathrm{PDF}$
}

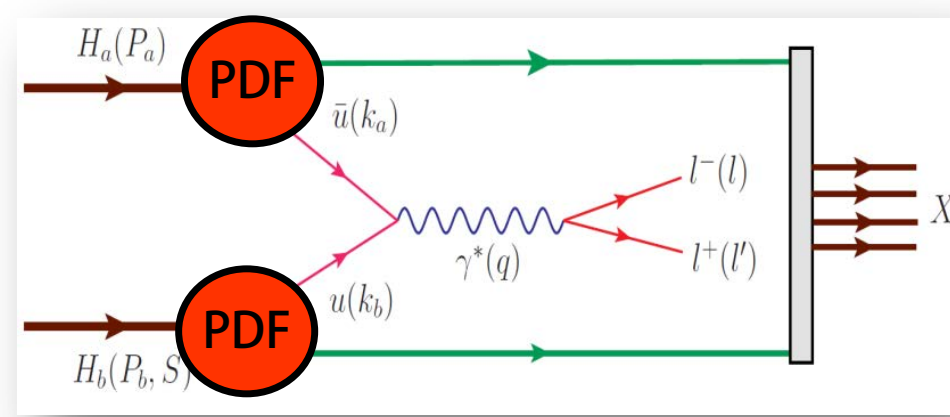

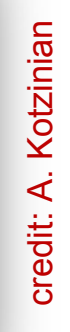

\section{Probe Universality}

are TMD PDFs in SIDIS identical to TMD PDFs in DY?

Test using unpolarized experiments, transverse SSA and DSA 


\section{LO SIDIS and single polarized DY cross sections}

\section{SIDIS}

$$
\begin{aligned}
& \frac{d \sigma_{\text {SIDIS }}^{L O}}{d x d y d z d p_{T}^{2} d \varphi_{h} d \psi}=\left[\frac{\alpha}{x y Q^{2}} \frac{y^{2}}{2(1-\varepsilon)}\left(1+\frac{\gamma^{2}}{2 x}\right)\right] \\
& \times\left(F_{U U, T}+\varepsilon F_{U U, L}\right)\left\{1+\cos 2 \phi_{h}\left(\varepsilon A_{U U}^{\cos 2 \phi_{h}}\right)\right. \\
& +\mathrm{S}_{\mathrm{T}}\left[\begin{array}{l}
\sin \left(\phi_{h}-\phi_{S}\right)\left(A_{U T}^{\sin \left(\phi_{h}-\phi_{S}\right)}\right) \\
+\sin \left(\phi_{h}+\phi_{S}\right)\left(\varepsilon A_{U T}^{\sin \left(\phi_{h}+\phi_{S}\right)}\right) \\
+\sin \left(3 \phi_{h}-\phi_{S}\right)\left(\varepsilon A_{U T}^{\sin \left(3 \phi_{h}-\phi_{S}\right)}\right)
\end{array}\right]
\end{aligned}
$$

Measure magnitude of azimuthal modulations in cross section: "Single Spin Asymmetries"

$$
\begin{aligned}
& \frac{d \sigma^{L O}}{d \Omega}=\frac{\alpha_{e m}^{2}}{F q^{2}} F_{U}^{1}\left\{1+\cos ^{2} \theta+\sin ^{2} \theta \cos 2 \varphi_{C S} A_{U}^{\cos 2 \varphi_{C S}}\right. \\
& \left.+S_{T}\left[\begin{array}{l}
\left(1+\cos ^{2} \theta\right) \sin \varphi_{S} A_{T}^{\sin \varphi_{S}} \\
+\sin ^{2} \theta\left(\begin{array}{l}
\sin \left(2 \varphi_{C S}+\varphi_{S}\right) A_{T}^{\sin \left(2 \varphi_{C S}+\varphi_{S}\right)} \\
+\sin \left(2 \varphi_{C S}-\varphi_{S}\right) A_{T}^{\sin \left(2 \varphi_{C S}-\varphi_{S}\right)}
\end{array}\right)
\end{array}\right)\right\}
\end{aligned}
$$

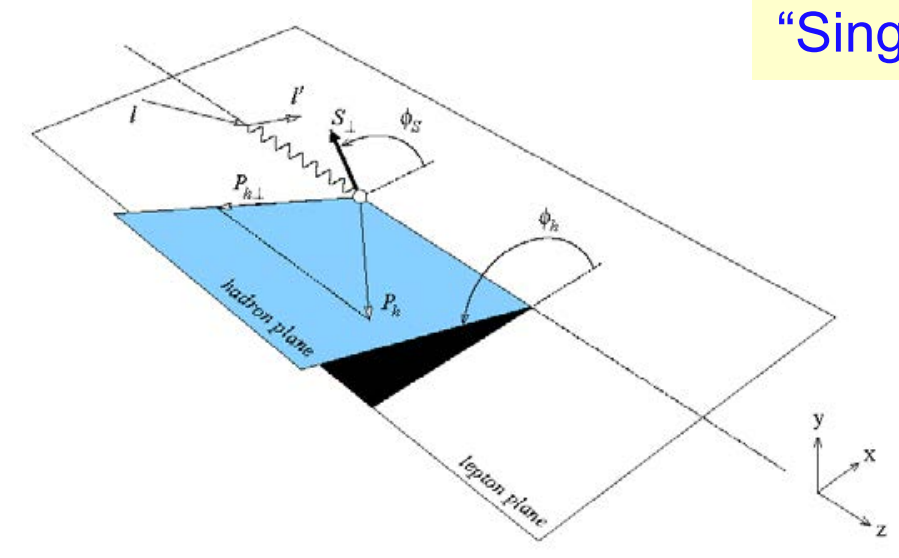

target rest frame

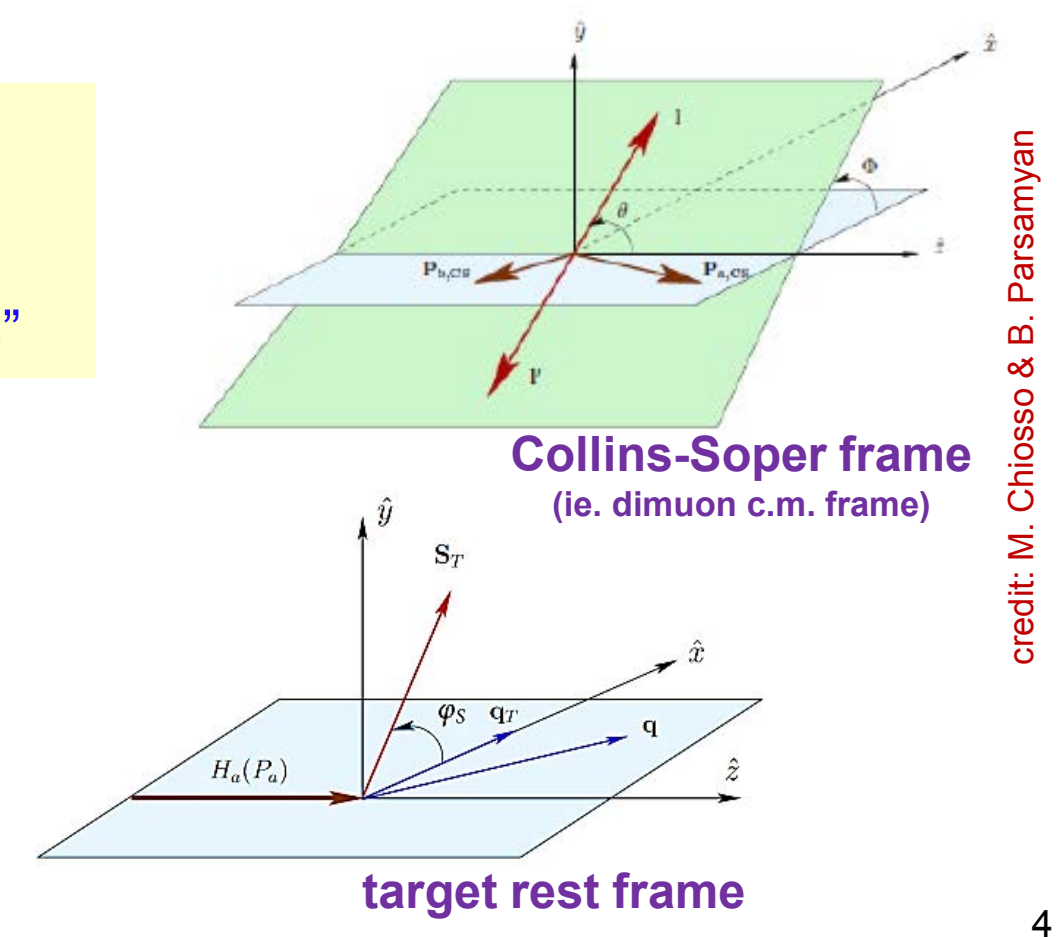




\section{LO SIDIS and single polarized DY cross sections}

\section{SIDIS}

$$
\begin{aligned}
& \frac{d \sigma_{S I D I S}^{L O}}{d x d y d z d p_{T}^{2} d \varphi_{h} d \psi}=\left[\frac{\alpha}{x y Q^{2}} \frac{y^{2}}{2(1-\varepsilon)}\left(1+\frac{\gamma^{2}}{2 x}\right)\right] \\
& \times\left(F_{U U, T}+\varepsilon F_{U U, L}\right)\left\{1+\cos 2 \phi_{h}\left(\varepsilon A_{U U}^{\cos 2 \phi_{h}}\right)\right.
\end{aligned}
$$

$\begin{array}{llllllll}A_{U U}^{\cos 2 \phi_{h}} & \propto & h_{1}^{\perp q} \otimes H_{1 q}^{\perp h} & & & & & \\ A_{U T}^{\sin \left(\phi_{h}-\phi_{s}\right)} & \propto & f_{1 T}^{\perp q} \otimes D_{1 q}^{h} & & \text { Sivers } \otimes & \text { FF } \\ A_{U T}^{\sin \left(\phi_{h}+\phi_{s}\right)} & \propto & h_{1}^{q} \otimes H_{1 q}^{\perp h} & \text { Transv } \otimes & \mathrm{CF} \\ A_{U T}^{\sin \left(3 \phi_{h}-\phi_{s}\right)} & \propto & h_{1 T}^{\perp q} \otimes H_{1 q}^{\perp h} & & \text { Pretz } \otimes & \mathrm{CF}\end{array}$

DY

$$
\begin{aligned}
& \frac{d \sigma^{L O}}{d \Omega}=\frac{\alpha_{e m}^{2}}{F q^{2}} F_{U}^{1}\left\{1+\cos ^{2} \theta+\sin ^{2} \theta \cos 2 \varphi_{C S} A_{U}^{\cos 2 \varphi_{C S}}\right. \\
& \left.+S_{T}\left[\begin{array}{l}
\left(1+\cos ^{2} \theta\right) \sin \varphi_{S} A_{T}^{\sin \varphi_{S}} \\
+\sin ^{2} \theta\left(\begin{array}{l}
\sin \left(2 \varphi_{C S}+\varphi_{S}\right) A_{T}^{\sin \left(2 \varphi_{C S}+\varphi_{S}\right)} \\
+\sin \left(2 \varphi_{C S}-\varphi_{S}\right) A_{T}^{\sin \left(2 \varphi_{C S}-\varphi_{S}\right)}
\end{array}\right)
\end{array}\right]\right\}
\end{aligned}
$$

beam target

$\mathrm{PDF} \otimes \mathrm{PDF}$

$\mathrm{BM} \otimes \mathrm{BM} \quad A_{T}^{\cos 2 \varphi_{c s}} \propto h_{1}^{\perp q} \otimes h_{1}^{\perp}$

$f_{1} \otimes$ Sivers $\quad A_{T}^{\sin \varphi_{s}} \propto f_{1}^{q} \otimes f_{1 T}^{\perp q}$

$\mathrm{BM} \otimes$ Transv $\quad A_{T}^{\sin \left(2 \varphi_{\varphi_{s}}-\varphi_{s}\right)} \propto h_{1}^{\perp q} \otimes h_{1 T}^{\perp q}$

$\mathrm{BM} \otimes$ Pretz $\quad A_{T}^{\sin \left(2 \varphi_{\text {cs }}+\varphi_{s}\right)} \propto h_{1}^{\perp q} \otimes h_{1}^{q}$

within QCD TMD framework:

$$
\begin{aligned}
& \left.h_{1}^{\perp q}\right|_{\text {SIDIS }}=-\left.h_{1}^{\perp q}\right|_{D Y} \\
& \left.f_{1 T}^{\perp q}\right|_{\text {SIDIS }}=-\left.f_{1 T}^{\perp q}\right|_{D Y}
\end{aligned}
$$

$$
\begin{aligned}
& \left.h_{1}^{q}\right|_{\text {SIDIS }}=\left.h_{1}^{q}\right|_{D Y} \\
& \left.h_{1 T}^{\perp q}\right|_{\text {SIDIS }}=\left.h_{1 T}^{\perp q}\right|_{D Y}
\end{aligned}
$$




\section{Drell Yan Advantage}

- Complementarity is emphasized by (LO): (Arnold,Metz,Schlegel:PRD79,034005(2009))

$\Rightarrow$ in SIDIS: there is $1 \mathrm{~F}_{\mathrm{U}(\mathrm{L}), \mathrm{T}}$ per TMD

$\Rightarrow$ in DY:

at least 2) $F_{(U) T}$ per TMD

$\rightarrow$ same TMDs can be measured in different $F_{(U) T}$

$\rightarrow$ allowing cross checks of TMD extraction

\& even of underlying formalism

$A_{U U}^{\cos 2 \phi_{h}} \propto h_{1}^{\perp q} \otimes H_{1 q}^{\perp h}$

$A_{U T}^{\sin \left(\phi_{h}-\phi_{s}\right)} \propto f_{1 T}^{\perp q} \otimes D_{1 q}^{h}$

$A_{U T}^{\sin \left(\phi_{h}+\phi_{s}\right)} \propto h_{1}^{q} \otimes H_{1 q}^{\perp h}$

$A_{U T}^{\sin \left(3 \phi_{h}-\phi_{s}\right)} \propto h_{1 T}^{\perp q} \otimes H_{1 q}^{\perp h}$
$\mathrm{PDF} \otimes \mathrm{FF}$

BM

Sivers $\otimes F F$

Transv $\otimes C F$

Pretz $\otimes C F$ beam target

$\mathrm{PDF} \otimes \mathrm{PDF}$

$B M \otimes B M$

$f_{1} \otimes$ Sivers

$B M \otimes$ Transv

BM $\otimes$ Pretz
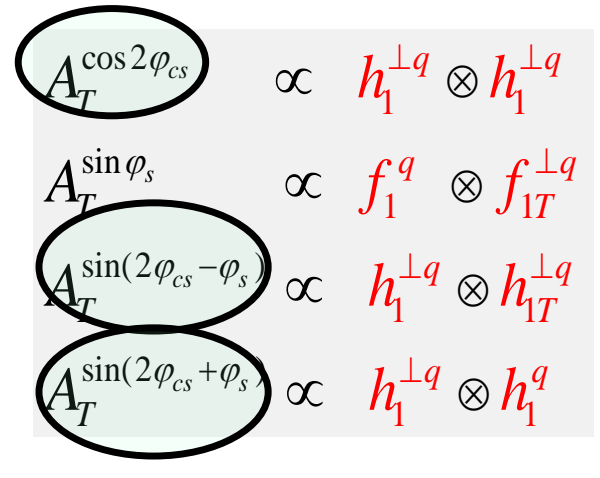

$$
A_{T}^{\sin \varphi_{s}}=\frac{F_{T}^{1}}{F_{U}^{1}}
$$




\section{Complementarity between SIDIS and Drell Yan}

- Complementarity is emphasized by (LO): (Arnold,Metz,Schlegel:PRD79,034005(2009))

$\Rightarrow$ in SIDIS: there is $1 \mathrm{~F}_{\mathrm{U}(\mathrm{L}), \mathrm{T}}$ per TMD

$\Rightarrow$ in DY: $\quad$ at least $2 \mathrm{~F}_{(\mathrm{U}) \mathrm{T}}$ per TMD

$\rightarrow$ same TMDs can be measured in different $F_{(U) T}$

$\rightarrow$ allowing cross checks of TMD extraction

\& even of underlying formalism TMD

- Systematic study of quark TMDs in Drell Yan

$\Rightarrow$ requires double-polarization

$\Rightarrow$ only then can all 8 leading twist TMD be measured

- Double-Spin Drell Yan

$\Rightarrow$ Measure DY with both Beam and Target polarized

$\longrightarrow$ broad spin physics program possible

$\longrightarrow$ truly complementary to spin physics programs at Jlab and RHIC 


\section{(Un)Polarized Drell Yan Experiments}

\begin{tabular}{|c|c|c|c|c|c|c|c|c|}
\hline Experiment & Particles & $\begin{array}{l}\text { Energy } \\
(\mathrm{GeV})\end{array}$ & $x_{b}$ or $x_{t}$ & $\begin{array}{l}\text { Luminosity } \\
\left(\mathrm{cm}^{-2} \mathrm{~s}^{-1}\right)\end{array}$ & $A_{T}^{\sin \phi_{S}}$ & $P_{b}$ or $P_{t}(f)$ & $\mathrm{rFOM}^{\#}$ & Timeline \\
\hline $\begin{array}{l}\text { COMPASS } \\
\text { (CERN) }\end{array}$ & $\pi^{-}+p^{\uparrow}$ & $\begin{array}{l}160 \mathrm{GeV} \\
\sqrt{\mathrm{s}}=17\end{array}$ & $x_{t}=0.1-0.3$ & $2 \times 10^{33}$ & 0.14 & $\begin{array}{c}P_{t}=90 \% \\
f=0.22\end{array}$ & $1.1 \times 10^{-3}$ & $\begin{array}{c}\text { 2015-2016 } \\
2018\end{array}$ \\
\hline $\begin{array}{l}\text { PANDA } \\
\text { (GSI) }\end{array}$ & $\overline{\mathbf{p}}+\mathbf{p}^{\uparrow}$ & $\begin{array}{l}15 \mathrm{GeV} \\
\sqrt{s}=5.5\end{array}$ & $x_{t}=0.2-0.4$ & $2 \times 10^{32}$ & 0.07 & $\begin{array}{c}P_{t}=90 \% \\
f=0.22\end{array}$ & $1.1 \times 10^{-4}$ & $>2024 ?$ \\
\hline $\begin{array}{l}\text { PAX } \\
\text { (GSI) }\end{array}$ & $\mathbf{p}^{\uparrow}+\bar{p}$ & $\begin{array}{l}\text { collider } \\
\sqrt{s}=14\end{array}$ & $x_{b}=0.1-0.9$ & $2 \times 10^{30}$ & 0.06 & $P_{b}=90 \%$ & $2.3 \times 10^{-5}$ & $>2020 ? ?$ \\
\hline $\begin{array}{l}\text { NICA } \\
\text { (JINR) }\end{array}$ & $p^{\uparrow}+p$ & $\begin{array}{l}\text { collider } \\
\sqrt{s}=26\end{array}$ & $x_{b}=0.1-0.8$ & $1 \times 10^{31}$ & 0.04 & $P_{b}=70 \%$ & $6.8 \times 10^{-5}$ & $>2023 ?$ \\
\hline $\begin{array}{l}\text { J-PARC } \\
\text { (high-p beam line) }\end{array}$ & $\pi^{-}+p$ & $\begin{array}{l}10-20 \mathrm{GeV} \\
\sqrt{s}=4.4-6.2\end{array}$ & $\begin{array}{l}x_{b}=0.2-0.97 \\
x_{t}=0.06-0.6\end{array}$ & $2 \times 10^{31}$ & --- & --- & --- & $\begin{array}{c}>2019 ? \\
\text { under discussion }\end{array}$ \\
\hline $\begin{array}{l}\text { fSPHENIX } \\
\text { (RHIC) }\end{array}$ & $\mathbf{p}^{\uparrow}+\mathbf{p}^{\uparrow}$ & $\begin{array}{l}\sqrt{s}=200 \\
\sqrt{s}=510\end{array}$ & $\begin{array}{c}x_{b}=0.1-0.5 \\
x_{b}=0.05-0.6\end{array}$ & $\begin{array}{l}8 \times 10^{31} \\
6 \times 10^{32}\end{array}$ & 0.08 & $\begin{array}{l}P_{b}=60 \% \\
P_{b}=50 \%\end{array}$ & $\begin{array}{l}4.0 \times 10^{-4} \\
2.1 \times 10^{-3}\end{array}$ & $>2021 ?$ \\
\hline $\begin{array}{l}\text { SeaQuest } \\
\text { (FNAL: E-906) }\end{array}$ & $p+p$ & $\begin{array}{l}120 \mathrm{GeV} \\
\sqrt{\mathrm{s}}=15\end{array}$ & $\begin{array}{l}x_{b}=0.35-0.9 \\
x_{t}=0.1-0.45\end{array}$ & $3.4 \times 10^{35}$ & --- & --- & --- & $2012-2017$ \\
\hline $\begin{array}{l}\text { Pol tgt DY } \\
\text { (FNAL: E-1039) }\end{array}$ & $\begin{array}{l}p+p^{\uparrow} \\
p+d^{\uparrow}\end{array}$ & $\begin{array}{l}120 \mathrm{GeV} \\
\sqrt{\mathrm{s}}=15\end{array}$ & $x_{t}=0.1-0.45$ & $\begin{array}{l}3.0 \times 10^{35} \\
3.5 \times 10^{35}\end{array}$ & $\begin{array}{c}0- \\
0.2^{*}\end{array}$ & $\begin{array}{c}P_{t}=85 \% \\
f=0.176\end{array}$ & 0.15 & 2018-2020 \\
\hline $\begin{array}{l}\text { Pol beam DY§ } \\
\text { (FNAL: E-1027) }\end{array}$ & $\mathbf{p}^{\uparrow}+\mathbf{p}$ & $\begin{array}{l}120 \mathrm{GeV} \\
\sqrt{\mathrm{s}}=15\end{array}$ & $x_{b}=0.35-0.9$ & $2 \times 10^{35}$ & 0.04 & $P_{b}=60 \%$ & 1 & $>2021 ?$ \\
\hline
\end{tabular}




\section{COMPASS 2015 Results}

- COMPASS: $190 \mathrm{GeV} \pi^{-}$beam on transverse polarized $\mathrm{H}$ target $\left(\mathrm{NH}_{3}\right)$

$\Rightarrow$ first year of polarized running completed

$\Rightarrow 2015$ data $\sim 120$ days

$\Rightarrow$ Transverse target polarization $\sim 80 \%$

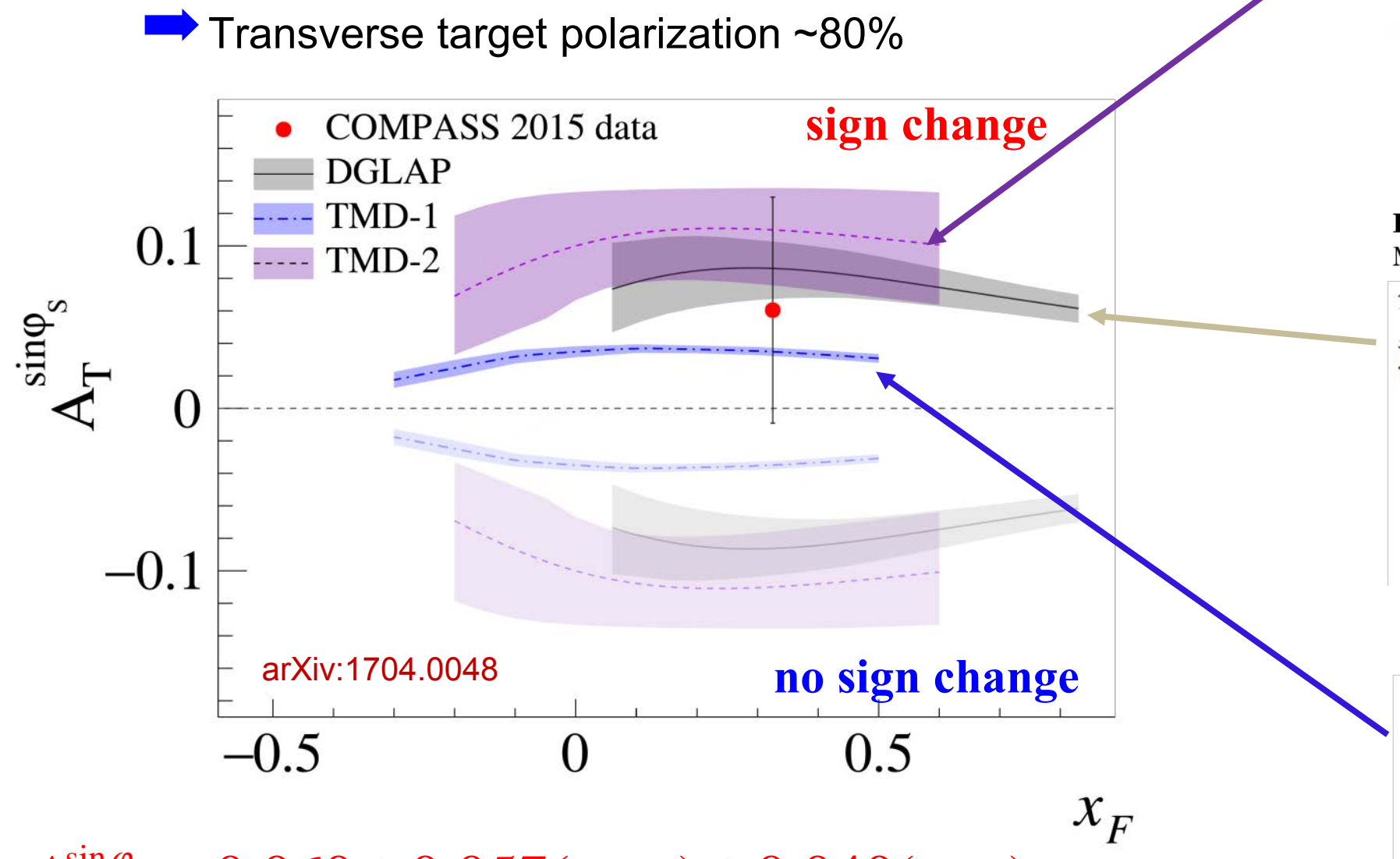

$A_{T}^{\sin \varphi_{s}}=0.060 \pm 0.057($ stat. $) \pm 0.040$ (sys.)

TMD-2 (2013)

P. Sun, F. Yuan, PRD88, 114012

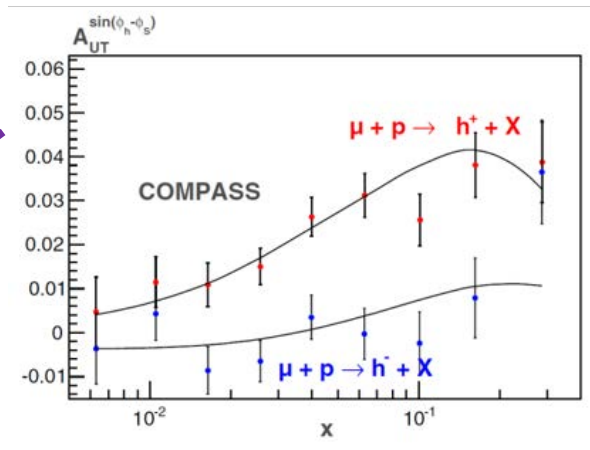

DGLAP (2016)

M. Anselmino et al., arXiv:1612.06413

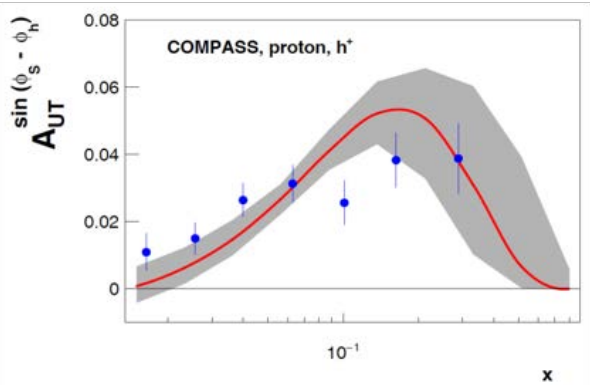

TMD-1 (2014)

M. G. Echevarria et al. PRD89,074013

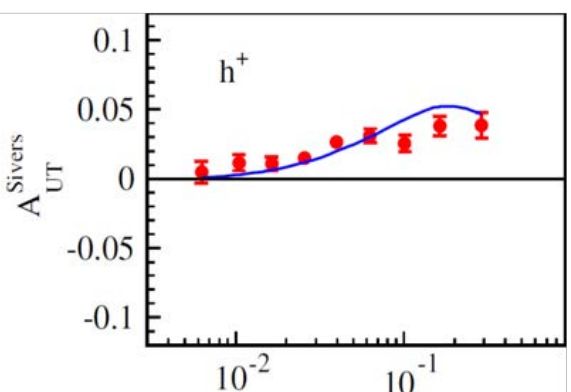




\section{COMPASS 2015 Results - II}

- Drell-Yan analysis performed in the mass range of $4.3-8.5 \mathrm{GeV} / \mathrm{c}^{2}$

$\Rightarrow$ Only $4 \%$ background in this mass range

$\Rightarrow$ DY events $\left[\mathrm{M}\left(\mu^{+} \mu^{-}\right)>4 \mathrm{GeV} / \mathrm{c}^{2}\right): \sim 35,000$

- Phase space for Drell-Yan and SIDIS partially overlap in the $x-Q^{2}$ plane

$\Rightarrow$ average $\mathrm{Q}^{2}$ in Drell-Yan is about $2 \mathrm{x}$ that in SIDIS

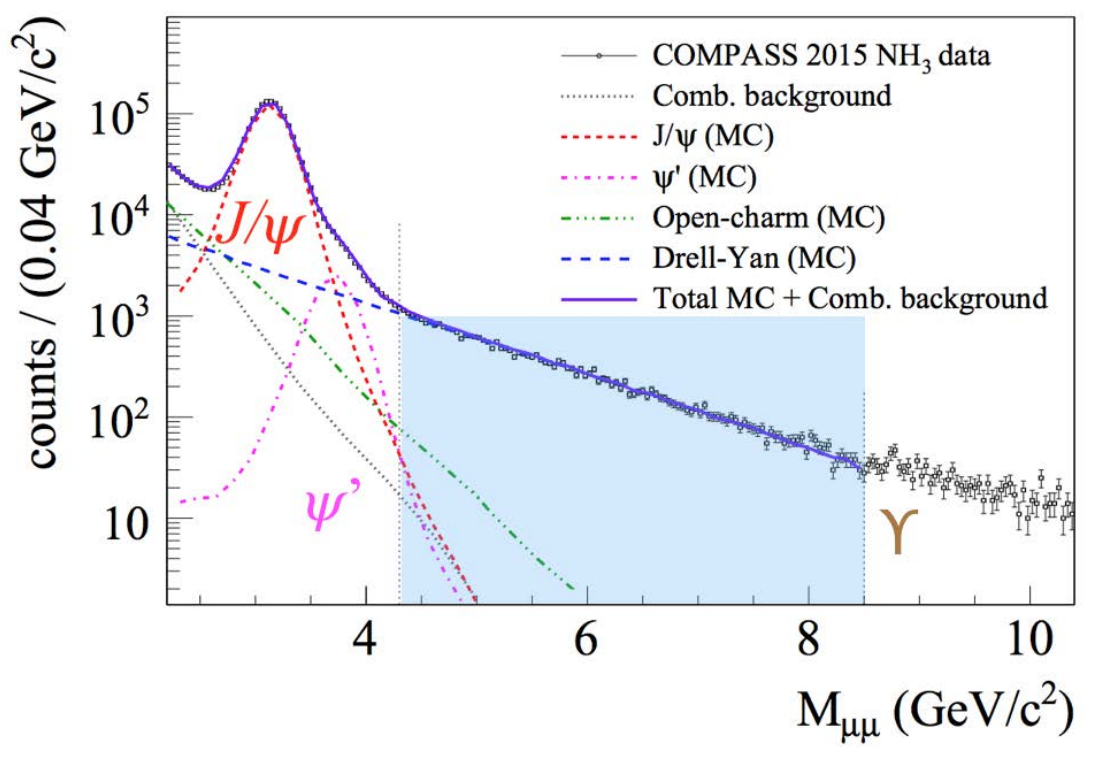

$\Rightarrow$ allows to minimize the impact of uncertainties from TMD scale evolution

$\Rightarrow$ overlap in kinematic regions of COMPASS Drell-Yan and SIDIS data allows for direct comparisons of TMD amplitudes

- COMPASS probes proton's valence quarks in Drell-Yan and SIDIS

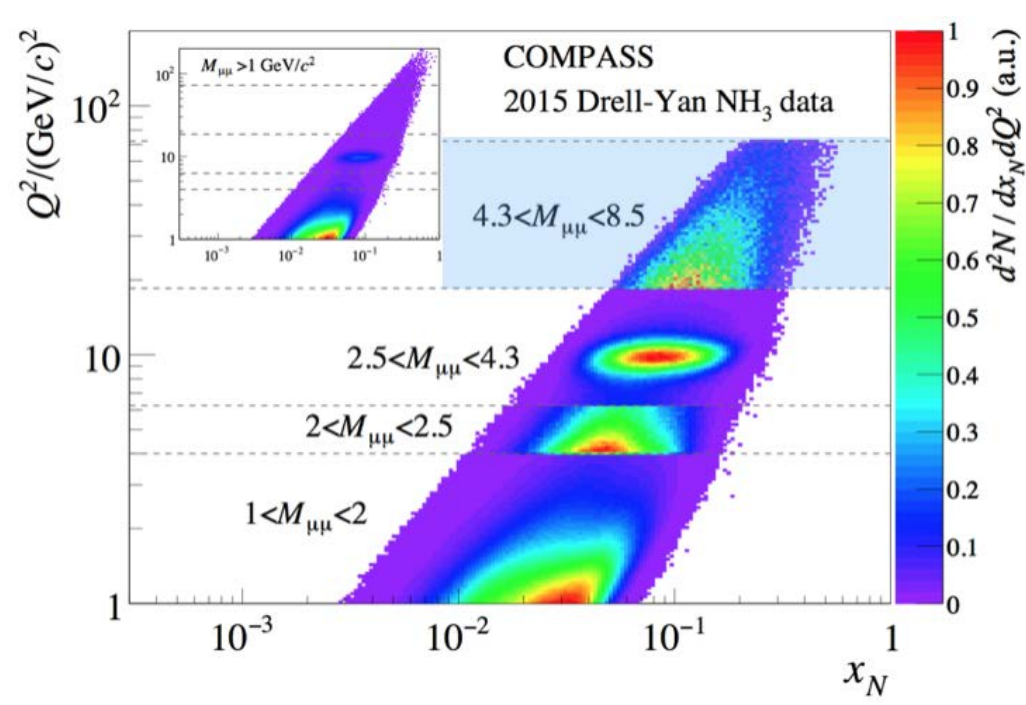




\section{COMPASS Plans}

- First physics results: April 2017

$\Rightarrow \sim 1 \sigma$ result

$\Rightarrow$ consistent w/ sign change!

- 2016-2017: DVCS program.

- 2018: second year of polarized DY planned

$\Rightarrow$ improved statistics expected

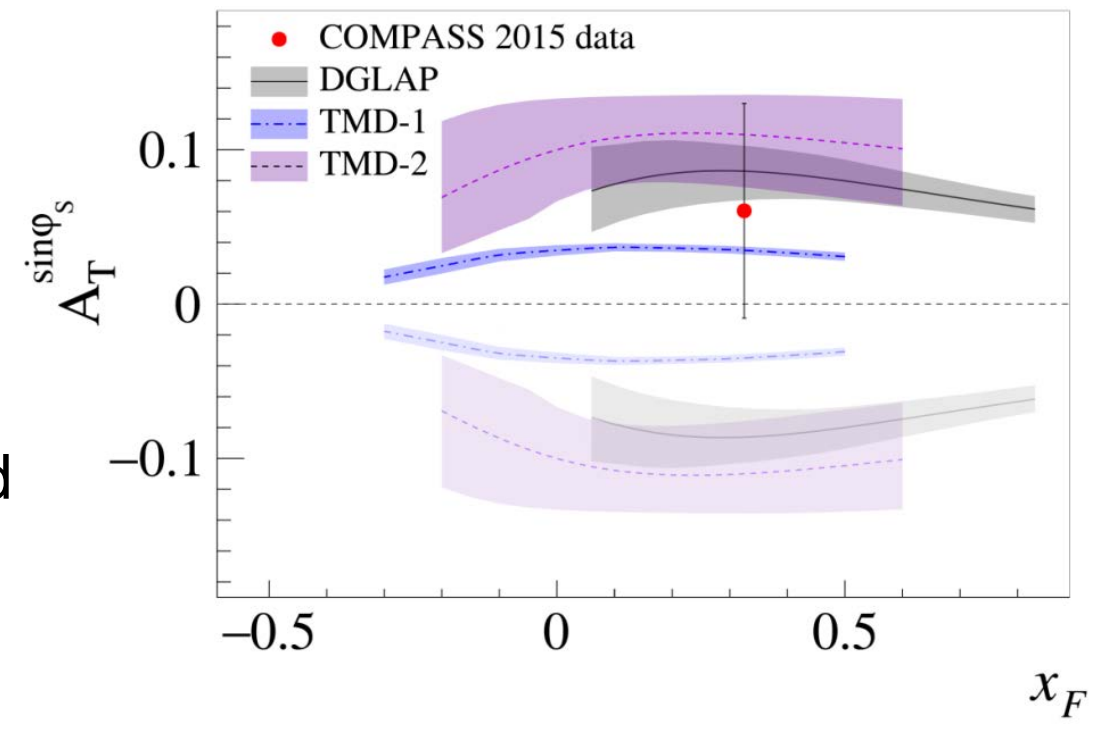

- COMPASS Beyond 2020 (under study: https://indico.cern.ch/event/502879/)

$\Rightarrow$ polarized ${ }^{6} \mathrm{LiD}$ target: flavor separation of TMD SSAs.

$\Rightarrow$ long $\mathrm{LH}_{2}$ and nuclei targets: un-polarized pion-induced DY

$\Rightarrow$ consider running with radio separated kaon/anti-proton beam for DY and spectroscopy

$\Rightarrow$ improve significantly our knowledge of pion and kaon PDFs

$\Rightarrow$ detailed study of the fundamental Lam-Tung relation violation

$\Rightarrow$ Gluon TMDs? 


\section{Current and Future DY Program at FNAL}

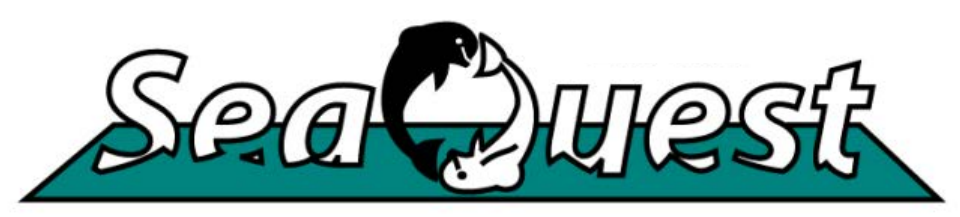

Unpolarized Beam and Target w/ SeaQuest detector

- E-906: $120 \mathrm{GeV}$ p from Main Injector on $\mathrm{LH}_{2}, \mathrm{LD}_{2}, \mathrm{C}, \mathrm{Fe}, \mathrm{W}$ targets $\rightarrow$ high-x Drell Yan

- Science run: March 2014 - July 2017

$\Rightarrow 2015$ data set: preliminary results

Polarized Beam and/or Target w/ SeaQuest detector

$\rightarrow$ development of high-Iuminosity facility for polarized Drell Yan

- E-1039: SeaQuest w/ pol $\mathrm{NH}_{3} / \mathrm{ND}_{3}$ targets (2018-2019)

$\Rightarrow$ probe sea quark distributions

- E-1027: pol p beam on (un)pol tgt (2020-2021?)

$\Rightarrow$ Sivers sign change (valence quark) 


\section{SeaQuest Spectrometer}

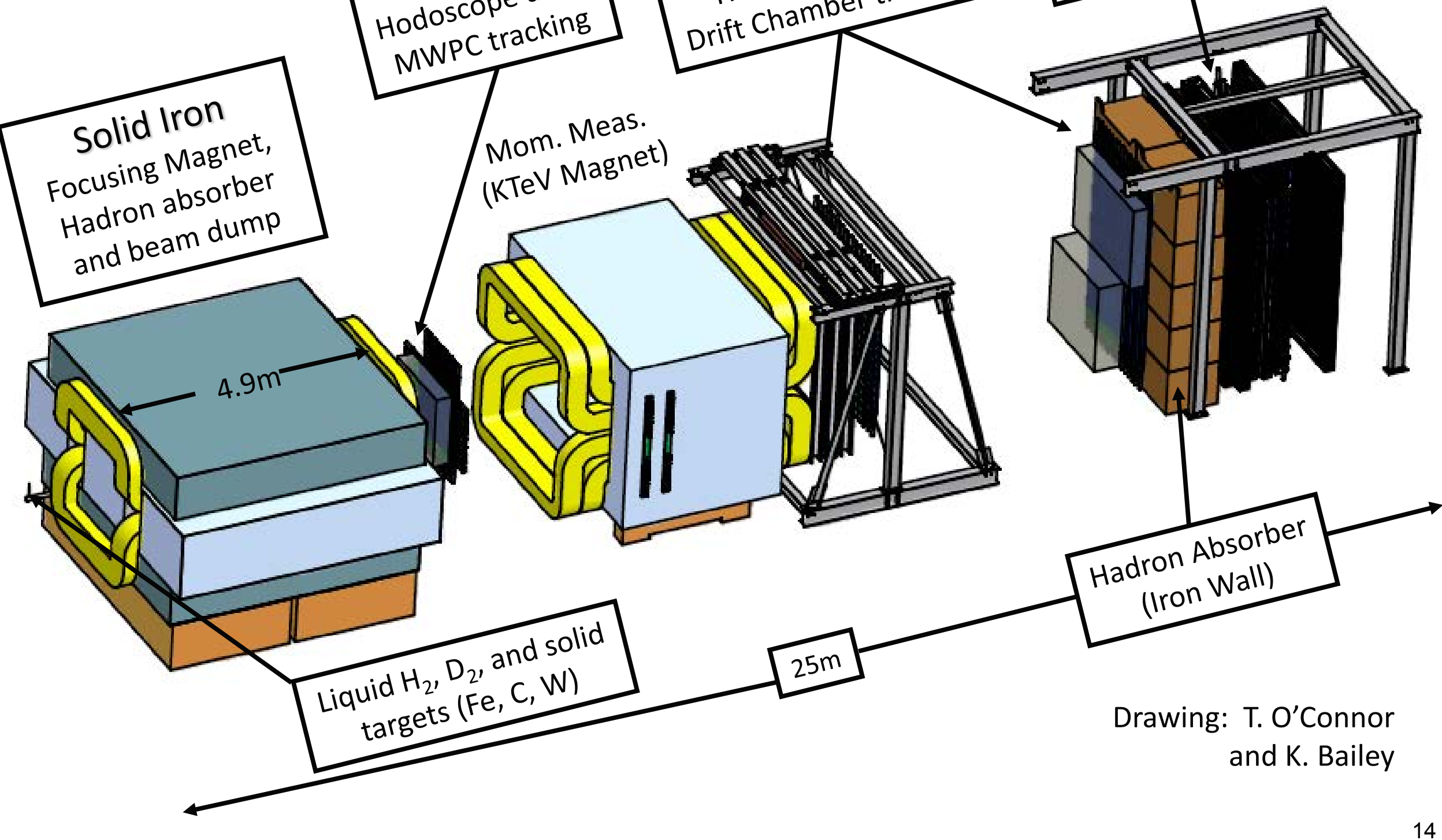




\section{Event Selection \& Reconstruction}
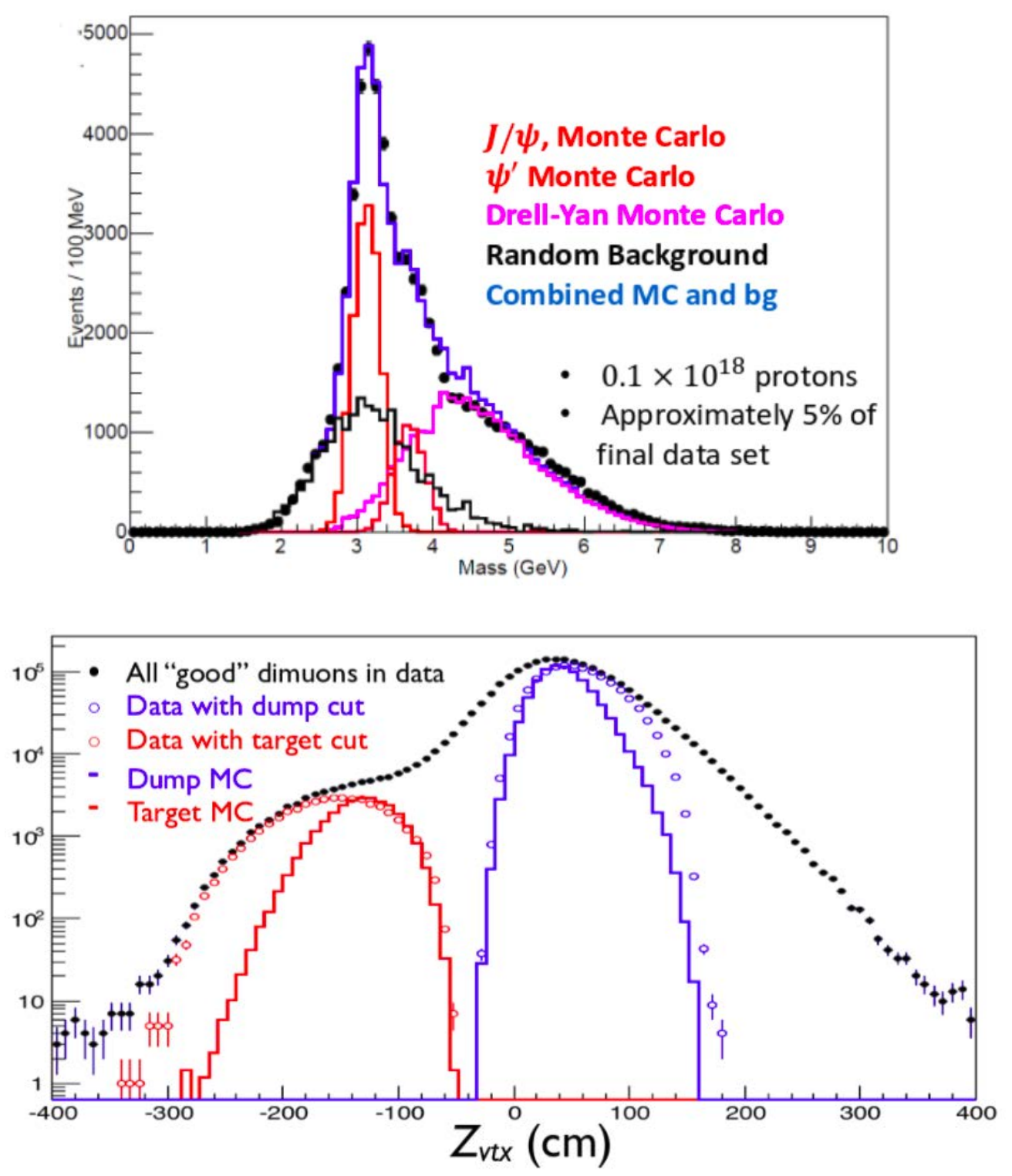

- Monte Carlo describe data well

- Resolution better than expected

- $\sigma_{\mathrm{M}}(\mathrm{J} / \psi) \sim 180 \mathrm{MeV}$

- $\sigma_{M}(D-Y) \sim 220 \mathrm{MeV}$

- J/ $\psi$ to $\psi^{\prime}$ separation

- lower J/ $\psi$ mass cut (more Drell-Yan events)

- good Target/Dump separation

- pointing resolution poor along beam axis

- dominated by random coincidences 


\section{Fixed Target Drell-Yan: Sensitivity to sea quarks}

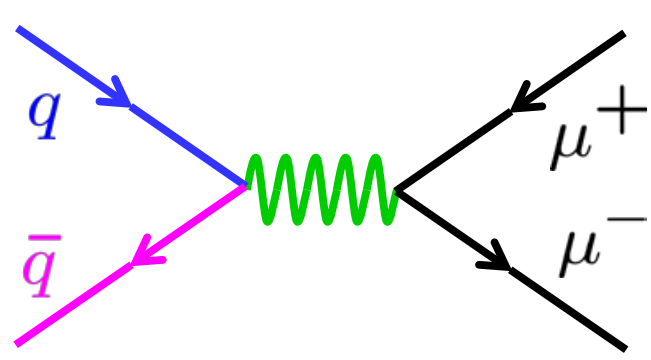

- Cross section: convolution of beam and target parton distributions

$\left.\frac{d^{2} \sigma}{d x_{b} d x_{t}}=\frac{4 \pi \alpha^{2}}{x_{b} x_{t} s} \sum_{q \in\{u, d, s, \ldots\}} e_{q}^{2} \bar{q}_{t}\left(x_{t}\right) q_{b}\left(x_{b}\right)+\bar{q}_{t}\left(x_{t}\right) \bar{q}_{b}\left(x_{b}\right)\right]$

u-quark dominance

$(2 / 3)^{2}$ vs. $(1 / 3)^{2}$ acceptance limited

(Fixed Target, Hadron Beam)

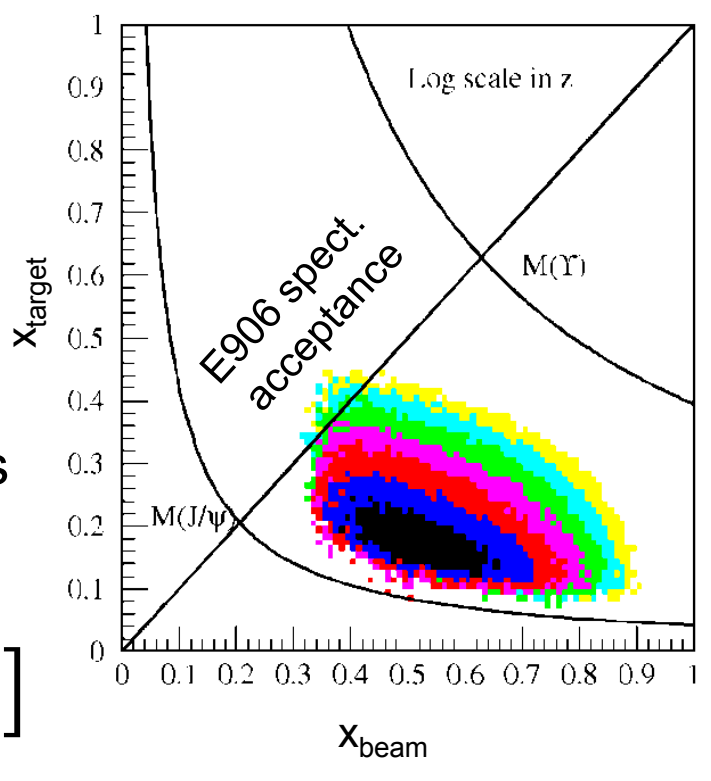

beam: valence quarks at high $\mathrm{x}$

target: sea quarks at low/intermediate $\mathrm{x}$

$$
\frac{\sigma^{\mathrm{pd}}}{2 \sigma^{\mathrm{pp}}}=\frac{1}{2}\left[1+\frac{\bar{d}(x)}{\bar{u}(x)}\right]
$$




\section{SeaQuest Cross Section Ratio (2015 Data Set)}

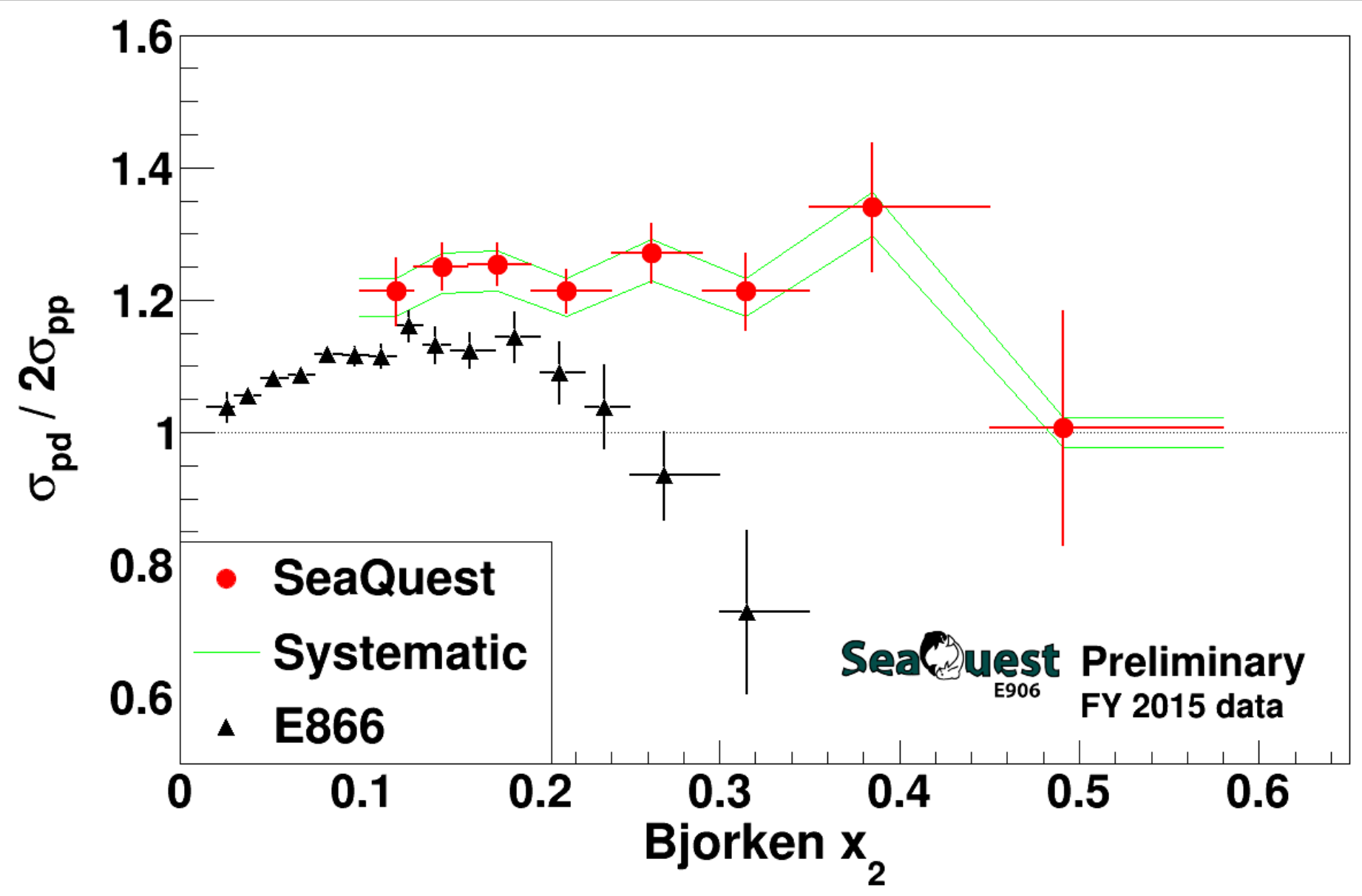

- different kinematics and $\mathrm{Q}^{2}$ for E866 \& SeaQuest data sets

- new chambers installed in March 2016: improve acceptance in high $x_{2}$ region

- $30 \%$ of anticipated data $\left(\sim 1.2 \times 10^{18}\right.$ pot $)$

- approved for $5 \times 10^{18}$ pot 


\section{SeaQuest Leading Order extraction (2015 Data Set)}

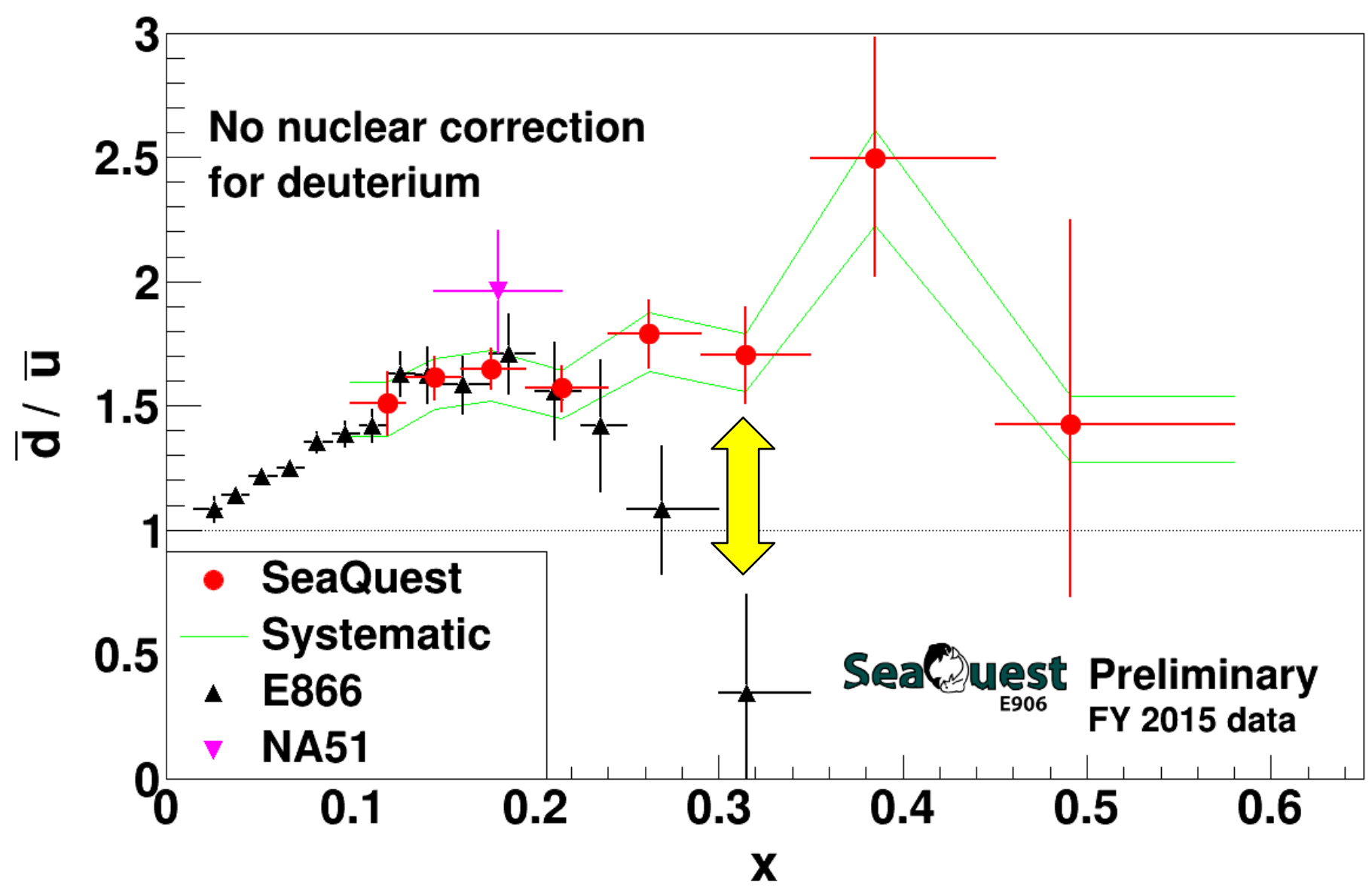

- E866 data is for $\mathrm{Q}^{2}=54 \mathrm{GeV}^{2}$ while SeaQuest data has $\mathrm{Q}^{2} \approx 29 \mathrm{GeV}^{2}$

- difference should be insignificant

- no nuclear correction for deuterium

- expected larger at higher $\mathrm{x}$, but still small compared to error bars

- is there disagreement at high $x$ ? 


\section{SeaQuest Leading Order extraction (2015 Data Set)}

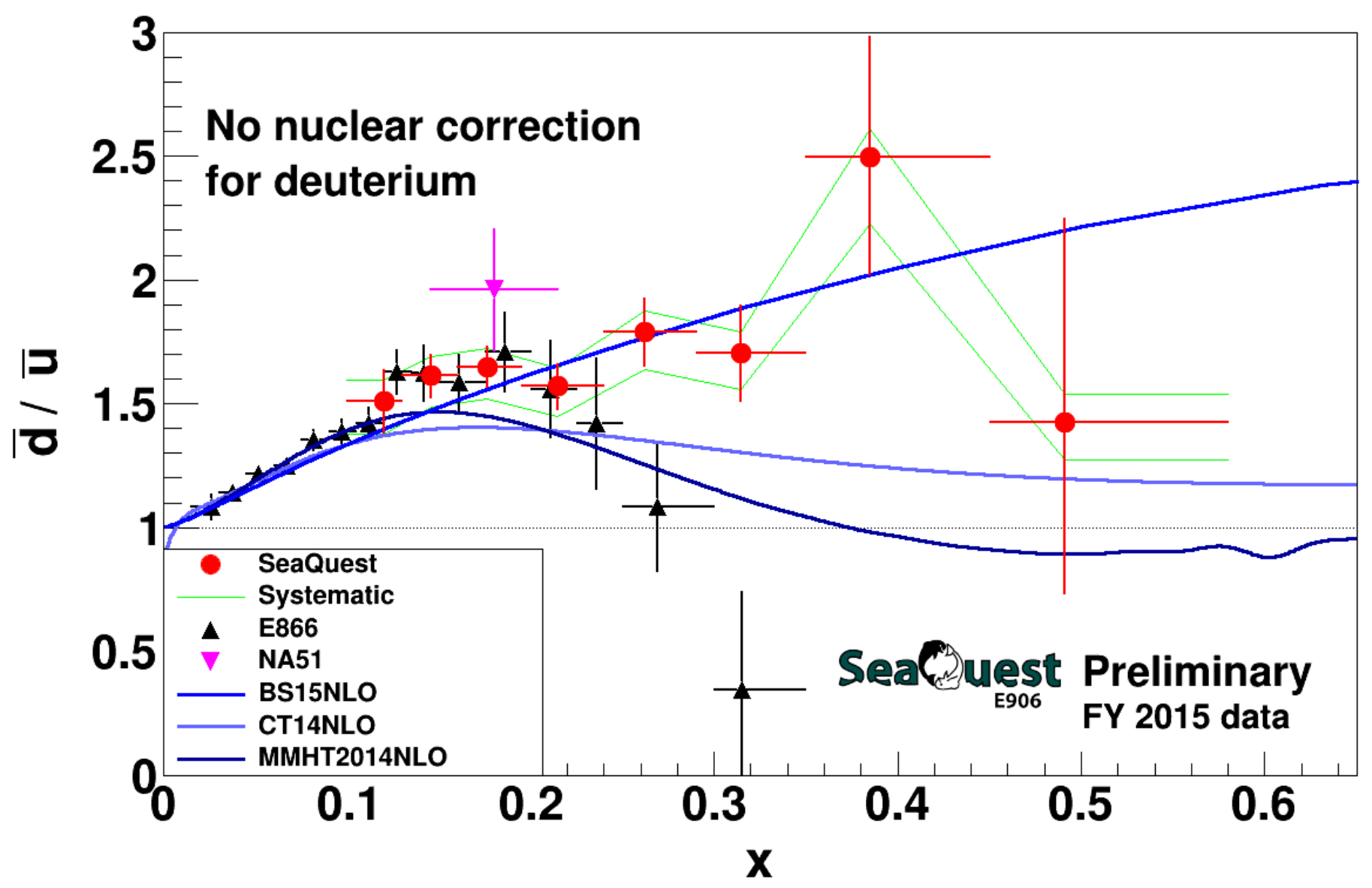

- BS15 (statistical model) calculated using parameters from NPA941(2015)307

- CT14 and MMHT2014 calculated with the LHAPDF library

- PDF scales taken as $29 \mathrm{GeV}^{2}$ 


\section{The Polarized Target}

- field: 5T @ 1K

- elliptical: $1.9 \mathrm{~cm} \times 2.1 \mathrm{~cm}(\mathrm{x}, \mathrm{y}), \mathrm{l}: 7.9 \mathrm{~cm}(\mathrm{z})$

- $\rho: 0.87 \mathrm{~g} / \mathrm{cm}^{3} \mathrm{NH}_{3}, 1 \mathrm{~g} / \mathrm{cm}^{3} \mathrm{ND}_{3}$

- packing fraction: 0.6

- dilution factor : $0.176,0.3$

- Polarization $<80 \%>,<32 \%>$

- IL: $8.6 \%, 9.5 \%$

- 3 active cells, 1 empty

- Helium consumption 100 l/day
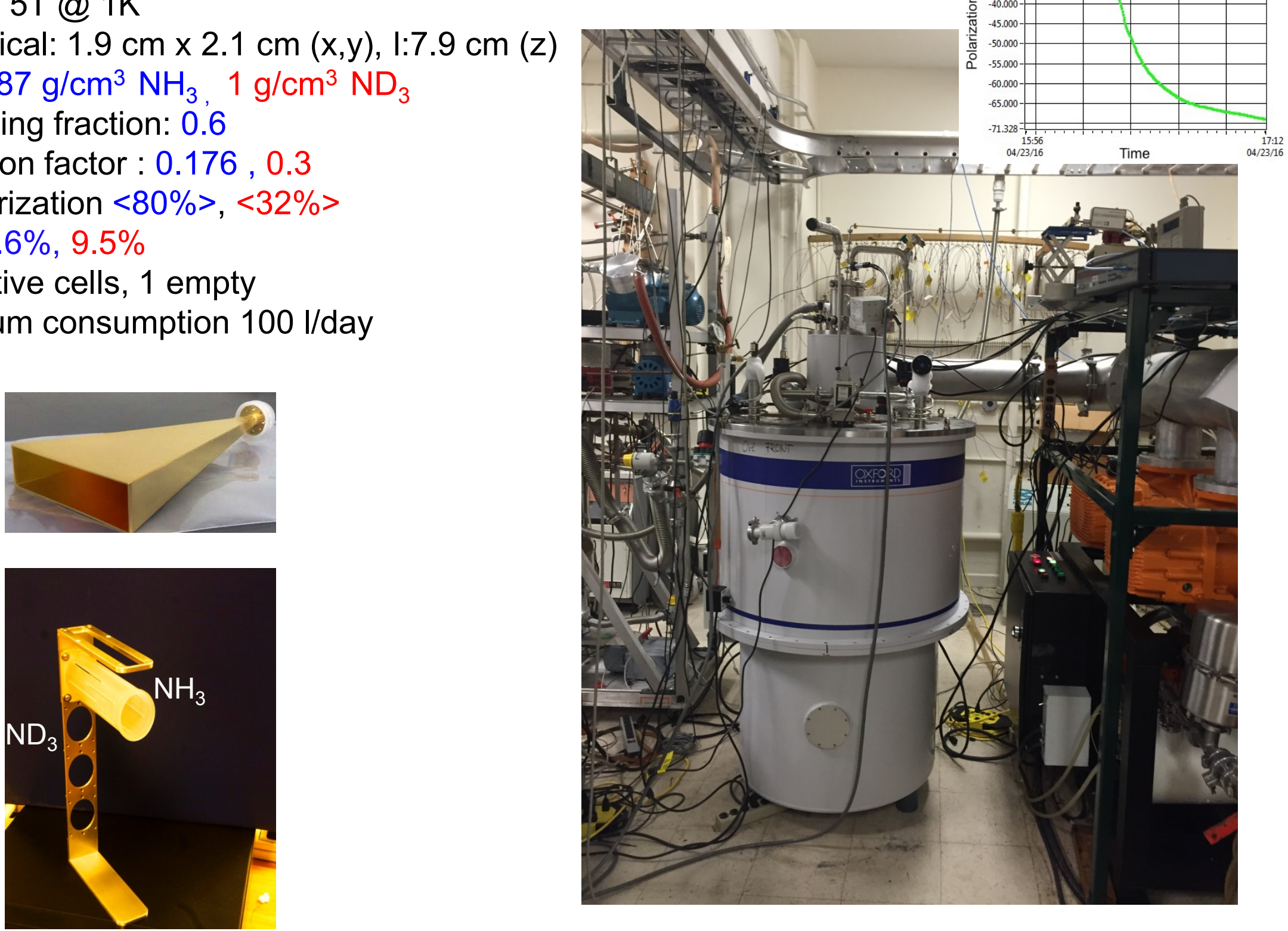


\section{The E1039 Target and FMAG}

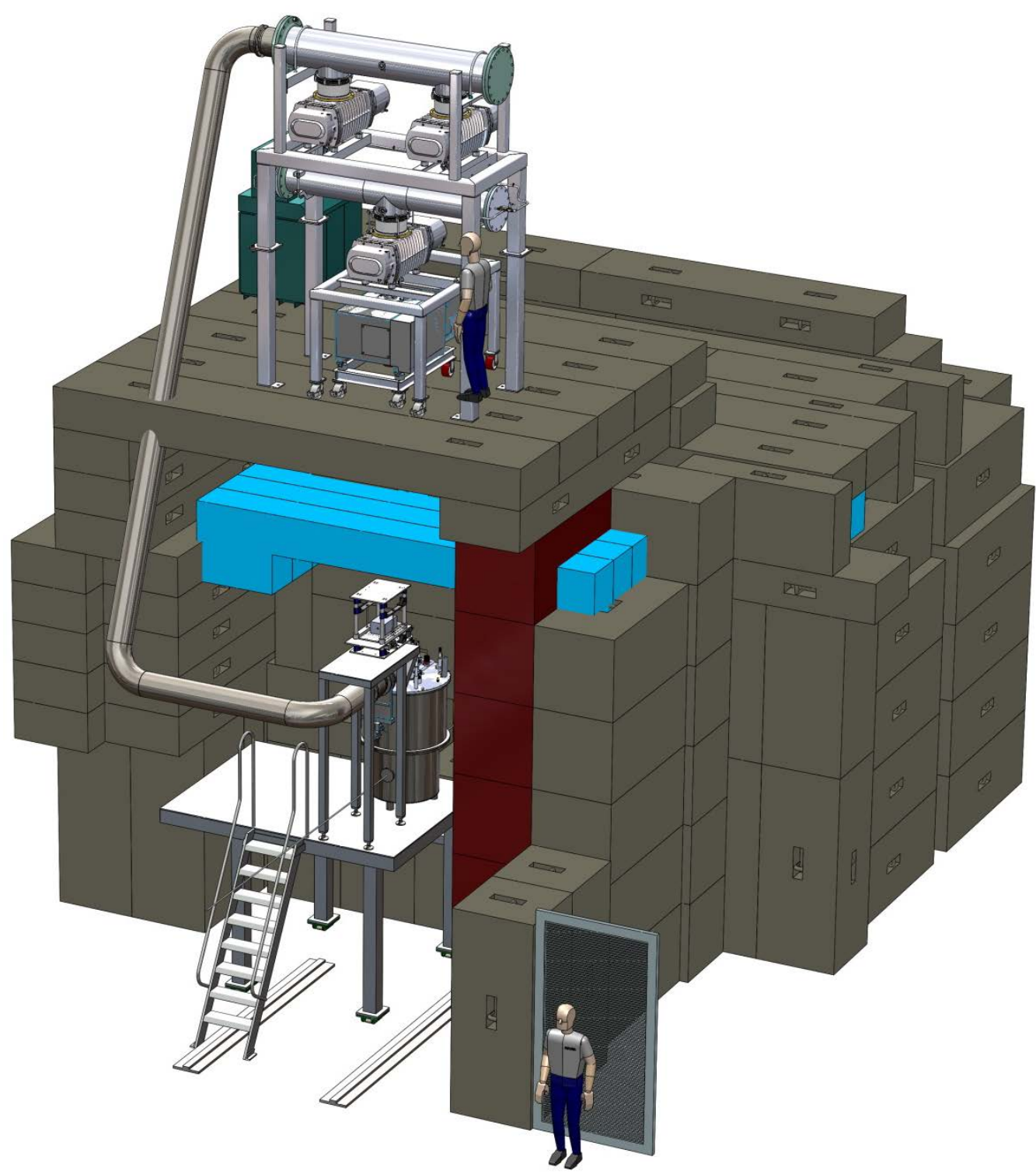

Changes needed:

- Collimators upstream

- Closed Loop He system

- 90 degree monitors L/R, T/B

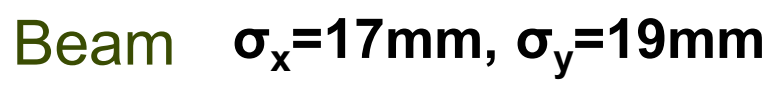

Target upstream by $\sim 200 \mathrm{~cm}$

- moves acceptance to lower $x_{2}$

- better target - dump separation 
$\mathrm{f}_{1 \mathrm{~T}}^{\perp}=\bigoplus_{\downarrow}-0$

\section{Sivers Function and Spin Crisis}

cannot exist w/o quark OAM

- describes transverse-momentum distribution of unpolarized quarks inside transversely polarized proton

- captures non-perturbative spin-orbit coupling effects inside a polarized proton

$$
\begin{array}{l|l}
\frac{1}{2}=\frac{1}{2} \Delta \Sigma+\Delta G+L & 1 / 2 \Delta \Sigma \approx 25 \% ; \Delta G \approx 20 \% \\
\Delta \Sigma=\Delta u+\Delta d+\Delta s & L \approx \text { unmeasured }
\end{array}
$$

\section{How measure quark OAM ?}

- GPD: Generalized Parton Distribution

- TMD: Transverse Momentum Distribution

$$
\begin{gathered}
A_{N}=\frac{N^{\uparrow}-N^{\downarrow}}{N^{\uparrow}+N^{\downarrow}} ? 0 \\
A_{N}^{D Y} \propto \frac{u\left(x_{b}\right) \cdot f_{1 T}^{\perp}, \bar{u}\left(x_{t}\right)}{u\left(x_{b}\right) \cdot \bar{u}\left(x_{t}\right)}
\end{gathered}
$$

Lattice QCD:

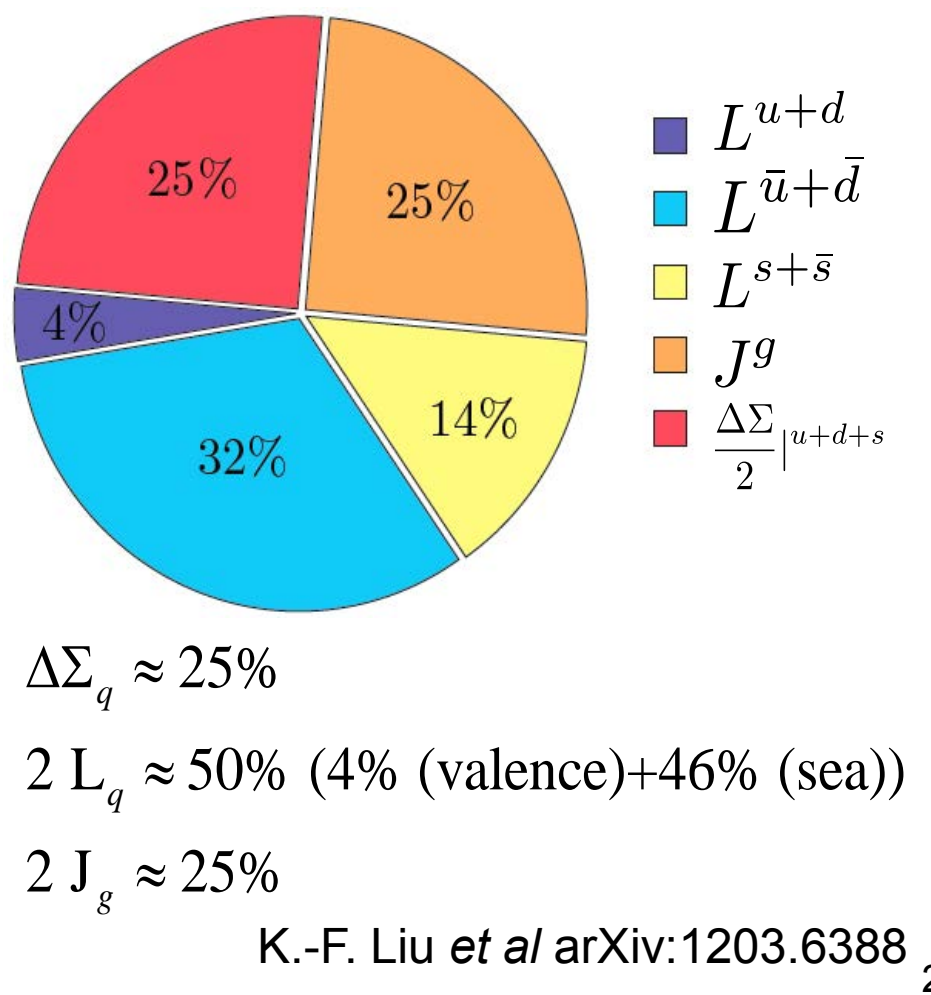




\section{Projected Statistical Precision with a Polarized Target at (E-1039)}

- Probe Sea Quark Sivers Asymmetry with a polarized proton/deuteron target at SeaQuest

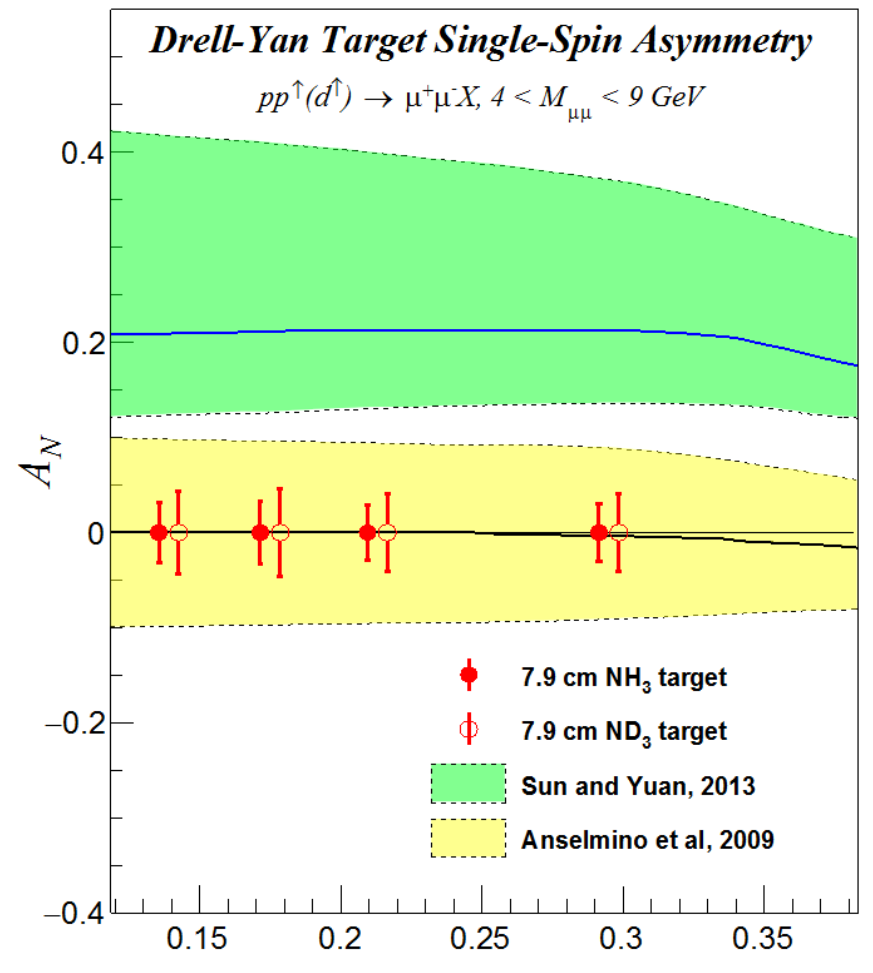

Statistics shown for two calendar years of running:
- existing SIDIS data poorly constrain sea-quark Sivers function (Anselmino)

- significant Sivers asymmetry expected from meson-cloud model (Sun \& Yuan)

- first Sea Quark Sivers Asymmetry Measurement

- determine sign and value of $\overline{\mathrm{u}}$ and $\overline{\mathrm{d}}$ Sivers distribution

If $A_{N} \neq 0$, major discovery:

"Smoking Gun" evidence for $\mathrm{L}_{\bar{u}, \bar{d}} \neq 0$

$$
A_{N}^{D Y}=\frac{2}{\pi} \cdot A_{T}^{\sin \varphi_{s}} \propto f_{1, u}^{q}\left(x_{b}\right) \otimes f_{1 T, \bar{u}(\bar{d})}^{\perp q}\left(X_{t}\right)
$$




\section{Tensor Polarization of Deuteron}

- deuteron is spin 1 particle, opens up new physics

spin-1 system in a B-field leads to 3 sublevels via Zeeman interaction Vector polarization: $\left(n^{+}-n^{-}\right)$; $-1<\mathrm{P}_{\mathrm{z}}<+1$ Tensor polarization: $\left(n^{+}-n^{0}\right)-\left(n^{0}-n^{-}\right) ;-2<P_{z z}<+1$

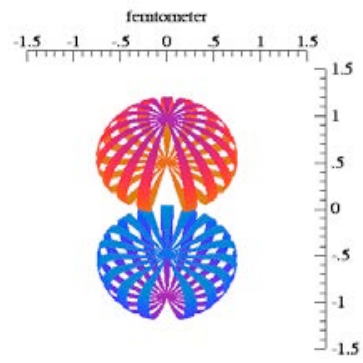

Normalization: $\left(n^{+}+n^{-}+n^{0}\right)=1$

Tensor structure $\mathbf{b}_{1}$ (e.g. deuteron) Tensor-structure crisis!?

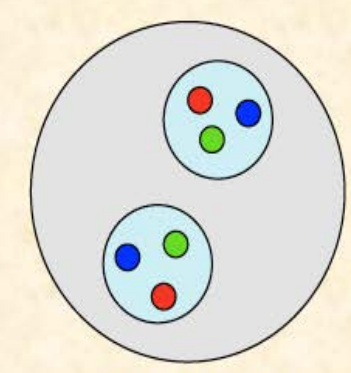

only $\mathrm{S}$ wave

$b_{1}=0$

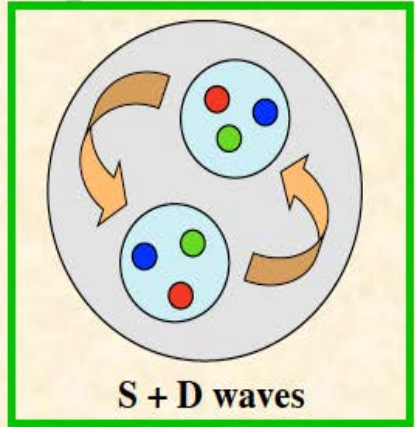

standard model $\mathbf{b}_{1} \neq \mathbf{0}$

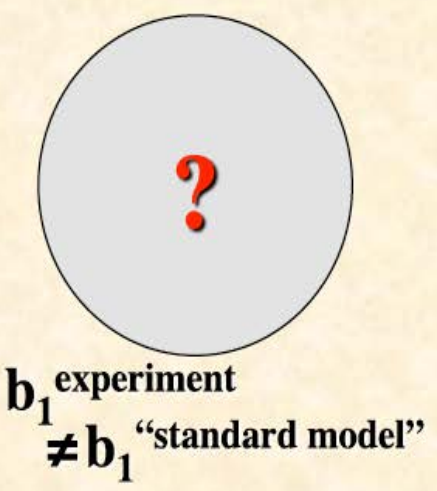

From S. Kumano, arxiv.org/1606.03149

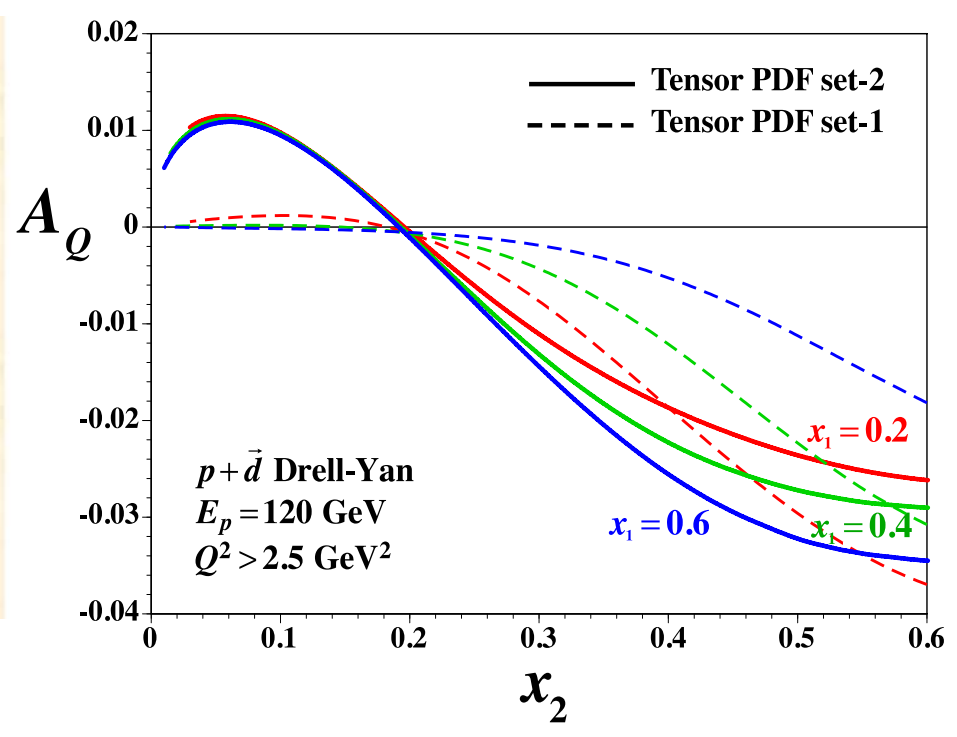




\section{Current Status and Plans for E-1039}

- Current status

$\Rightarrow$ full system cooldown/test with full extended $8 \mathrm{~cm}$ long target

$\Rightarrow$ reached $92 \%$ polarization

$\Rightarrow$ half of the liquefier system built and delivered, second half will be ordered late this year

$\Rightarrow$ beamline design $70 \%$ finished; now looking for reducing costs

$\Rightarrow$ currently working on $90 \%$ design of the whole installation and beam line

- Funding

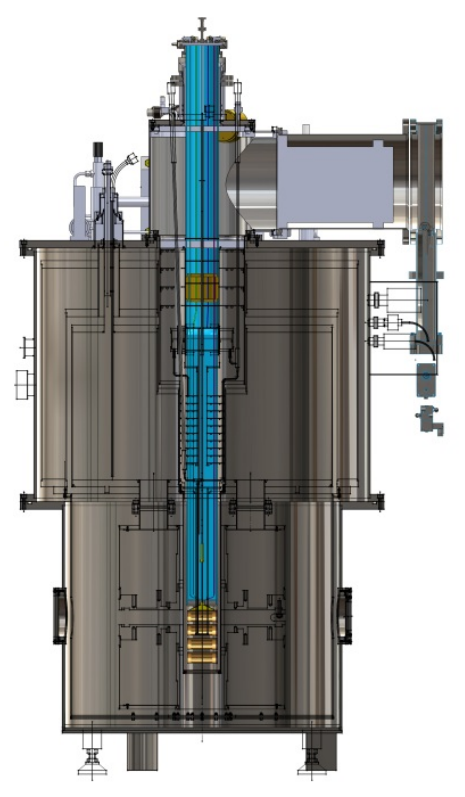

$\Rightarrow$ DoE has provided \$2 Mio for E-1039 in Sept 2017

$\Rightarrow$ Fermilab will pay to decommission E906 and to install E1039

- Plans

$\Rightarrow$ last system cooldown with both target sticks in Dec 2017

$\Rightarrow$ move target to FNAL in Jan 2018, system cooldown Feb 2018 at FNAL

$\Rightarrow$ start beam line commissioning in Mar 2018, and general commissioning in summer 2018

$\Rightarrow$ start data taking in fall 2018 


\section{Let's Polarize the Beam at Fermilab (E-1027)}

The Plan:

- Use fully understood SeaQuest Spectrometer

- Add polarized beam

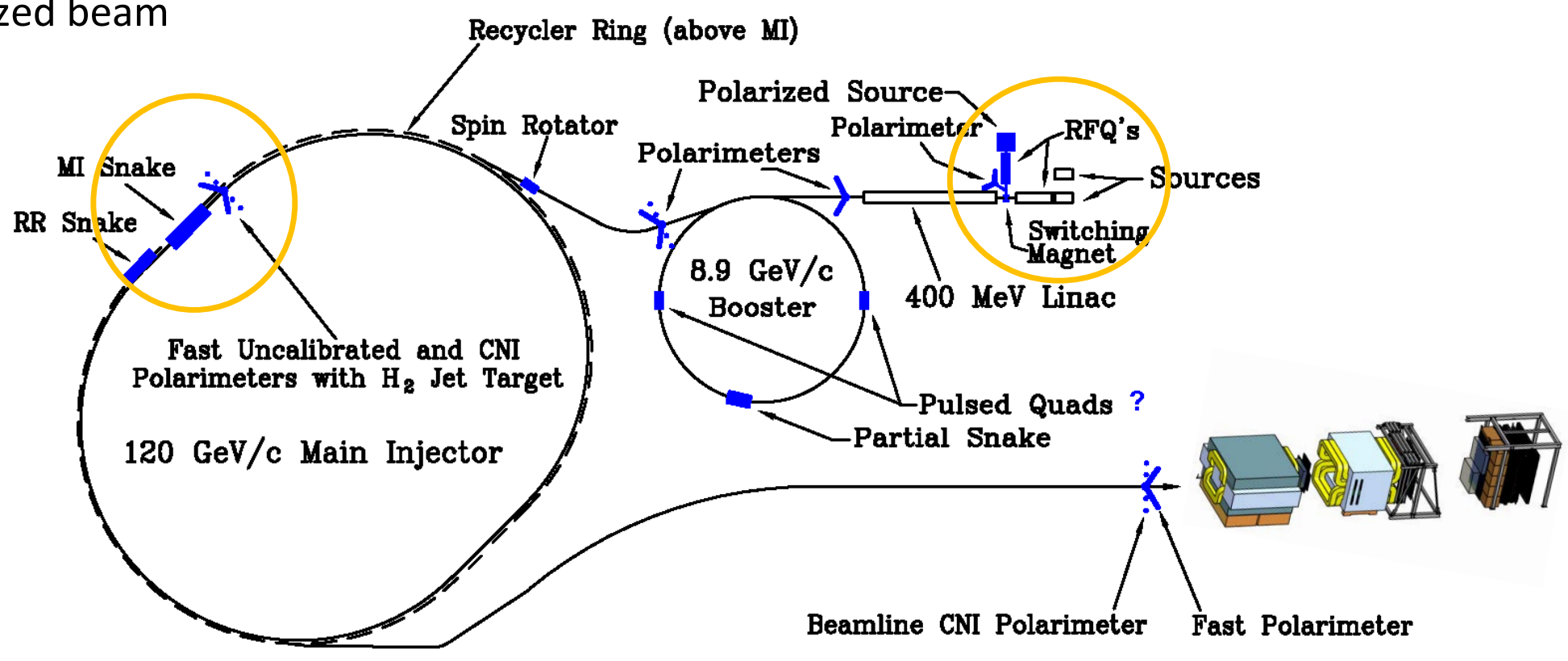

- Measure sign-change in Sivers Function:

$\longrightarrow$ QCD (and factorization) require sign change

$\longrightarrow$ major milestone in hadronic physics (HP13)

$$
\left.f \frac{\perp}{1 T}\right|_{S I D I S}=-\left.f \frac{\perp}{1 T}\right|_{D Y}
$$

- $\quad$ Fermilab (best place for polarized DY):

$\longrightarrow$ very high luminosity, large $x$-coverage (primary beam, fixed target)

- Cost Est.: $\$ 6 M+\$ 4 M$ Contingency \& Management $=\$ 10 M($ in 2013) 


\section{Expected Precision from E-1027 at Fermilab}

- Probe Valence Quark Sivers Asymmetry with a polarized proton beam at SeaQuest

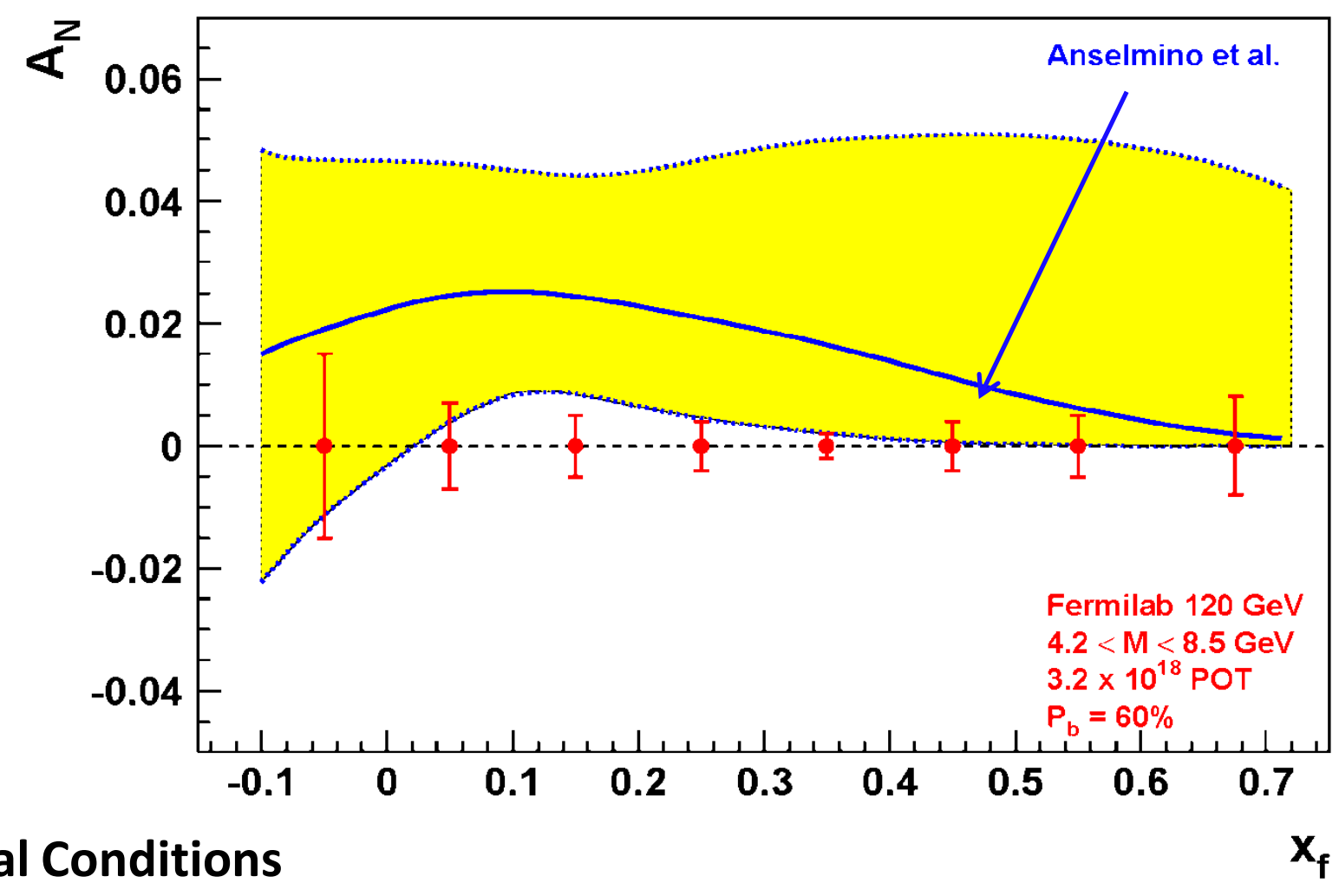

1.3 Mio DY events with no dilution

- Experimental Conditions

- same as SeaQuest

- luminosity: $\mathrm{L}_{\mathrm{av}}=2 \times 10^{35}$ (10\% of available beam time: $\left.\mathrm{I}_{\mathrm{av}}=15 \mathrm{nA}\right)$

- $\quad 3.2 \times 10^{18}$ total protons for $5 \times 10^{5} \mathrm{~min}$ : ( $=2 \mathrm{yrs}$ at $50 \%$ efficiency) with $\mathrm{P}_{\mathrm{b}}=60 \%$

Can measure not only sign, but also the size \& probably shape of the Sivers function! as well as TMD evolution! 


\section{Simulation of final polarization as function of Energy in MI}

- Simulations of final polarization as function of Energy in Fermilab Main Injector look promising (Meiqin Xiao (FNAL AD), Etienne Forest (KEK)):

$\Rightarrow$ point-like snake in correct location, $w /$ actual ramp rate for acceleration:

final polarization: 90\%

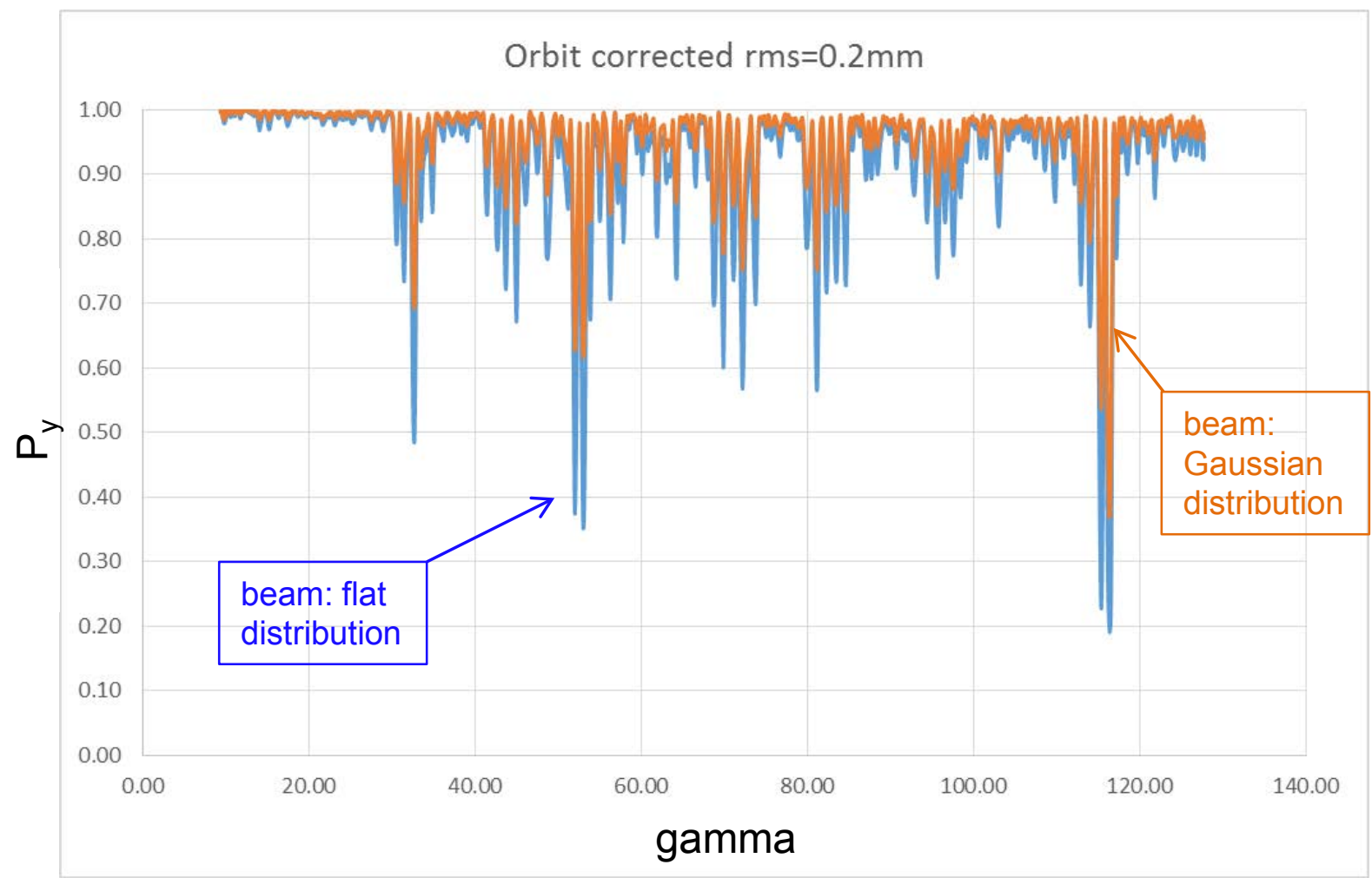

Polarizations with magnet field error and misalignment (from magnet database and survey group), corrected (for SeaQuest running conditions)

$\varepsilon_{\max }=20 \pi \mathrm{mm}$.mrad in y plane and $\Delta \mathrm{p}=1.25^{*} 10^{-3}$ in longitudinal plane 


\section{Polarized protons: Fermilab vs RHIC}

- Most significant difference:

Ramp time of Main Injector $<0.7 \mathrm{~s}$, at RHIC 1-2 min

$\Rightarrow$ warm magnets at MI vs. superconducting at RHIC

$\rightarrow$ pass through all depolarizing resonances much more quickly

- Beam remains in MI $\sim 2 \mathrm{~s}$, in RHIC $\sim 8$ hours

$\Rightarrow$ extracted beam vs. storage ring

$\Rightarrow$ much less time for cumulative depolarization

- Disadvantage compared to RHIC - no institutional history of accelerating polarized proton beams

$\Rightarrow$ Fermilab E704 had polarized beams through hyperon decays
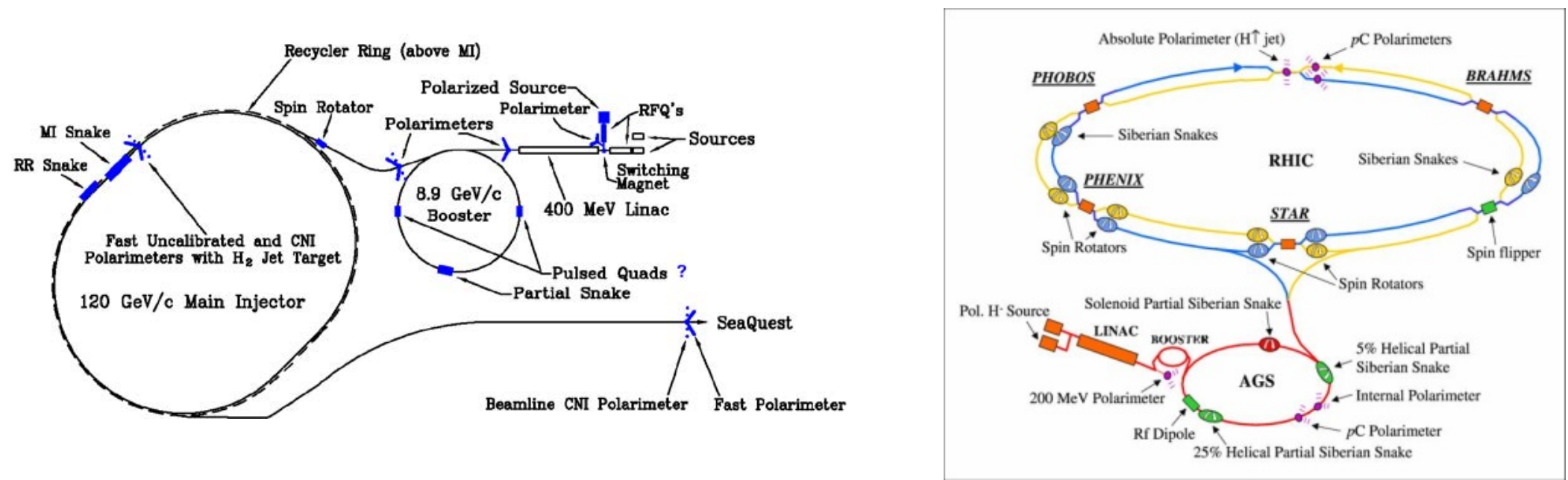


\section{J-PARC Projection \& Plans}

- Accessing GPD of nucleon via exclusive meson-induced Drell-Yan

$\Rightarrow$ Test of factorization of exclusive Drell-Yan process

$\Rightarrow$ Test of universality of GPD in space-like (DVMP) and time-like processes (DY).

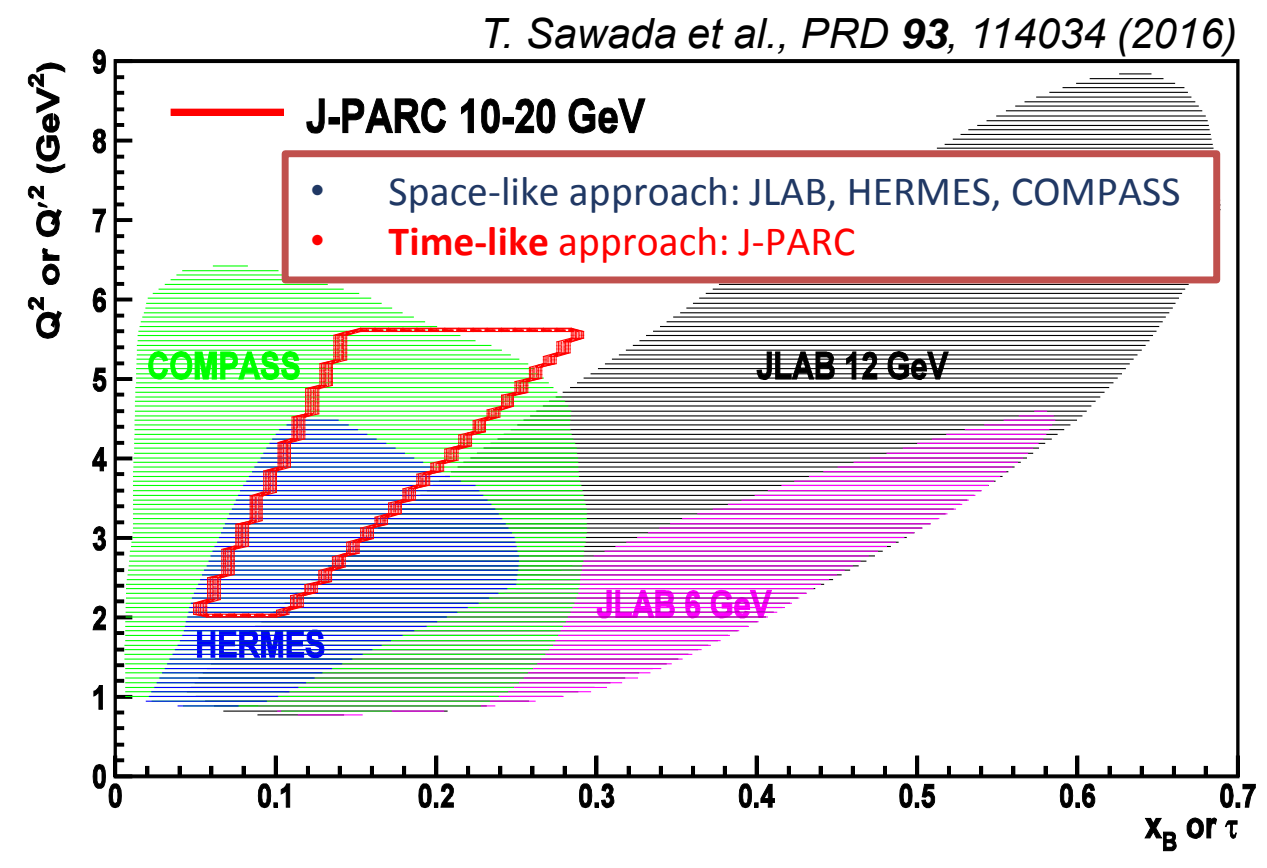

- E50 experiment (Stage-1 approved by J-PARC) + $\mu$-ID extension

$\Rightarrow$ 10-20 GeV $\pi^{-}$beam on high momentum beam line at J-PARC

$\Rightarrow$ good missing mass resolution in exclusive DY events $\left(\pi^{-} p \rightarrow \mu^{+} \mu^{-} n\right)$

$\Rightarrow$ Statistical accuracy adequate for discriminating between predictions from two current GPD models.

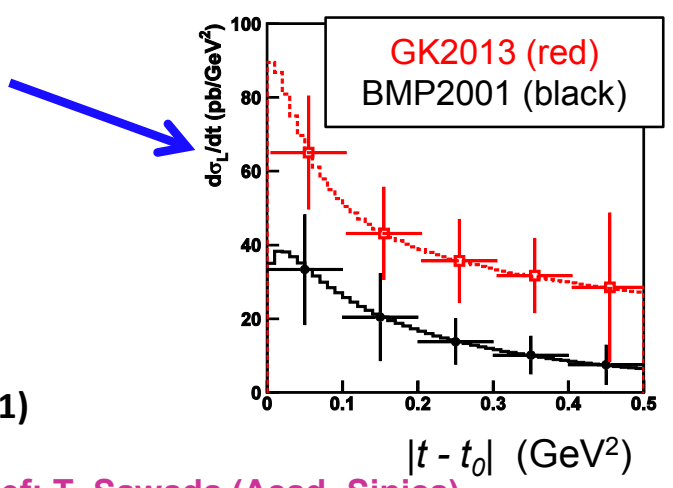

Ref: T. Sawada (Acad. Sinica)

$$
P_{\pi}=10 \mathrm{GeV}
$$

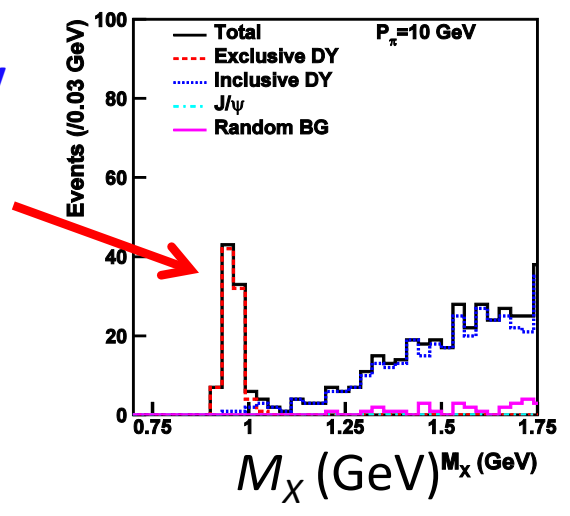




\section{Search for Dark Photons at SeaQuest}

- Classic Beam Dump Experiment

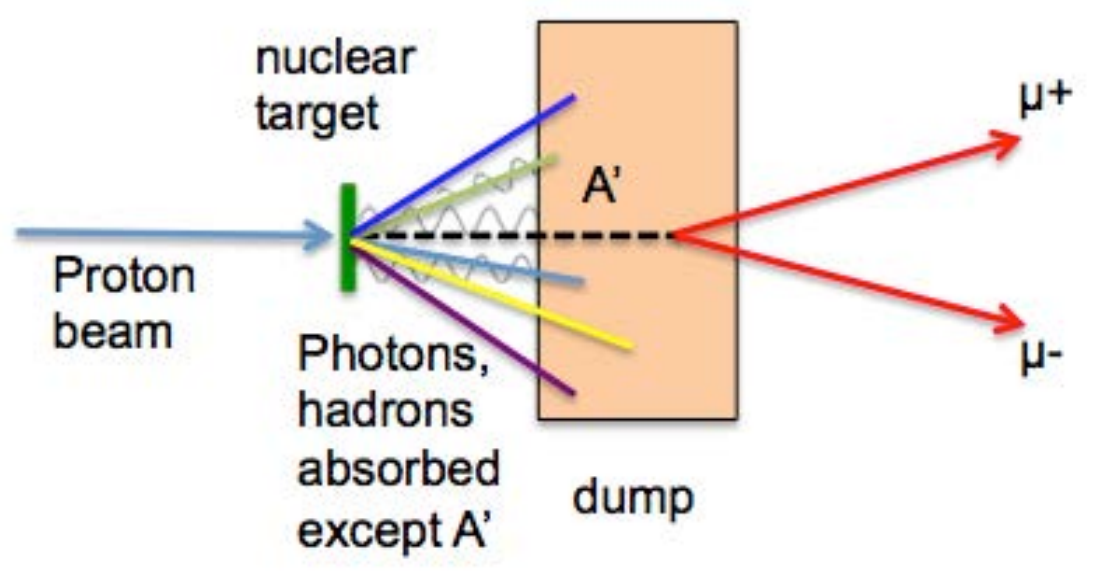

- Minimal impact on Drell-Yan program

$\Rightarrow$ run parasitically during E906

$l_{o} \approx \frac{0.8 \mathrm{~cm}}{N_{\text {eff }}}\left(\frac{E_{o}}{10 \mathrm{GeV}}\right)\left(\frac{10^{-4}}{\varepsilon}\right)^{2}\left(\frac{100 \mathrm{MeV}}{m_{A^{\prime}}}\right)^{2}$

J. D. Bjorken et al, PRD 80 (2009) 075018

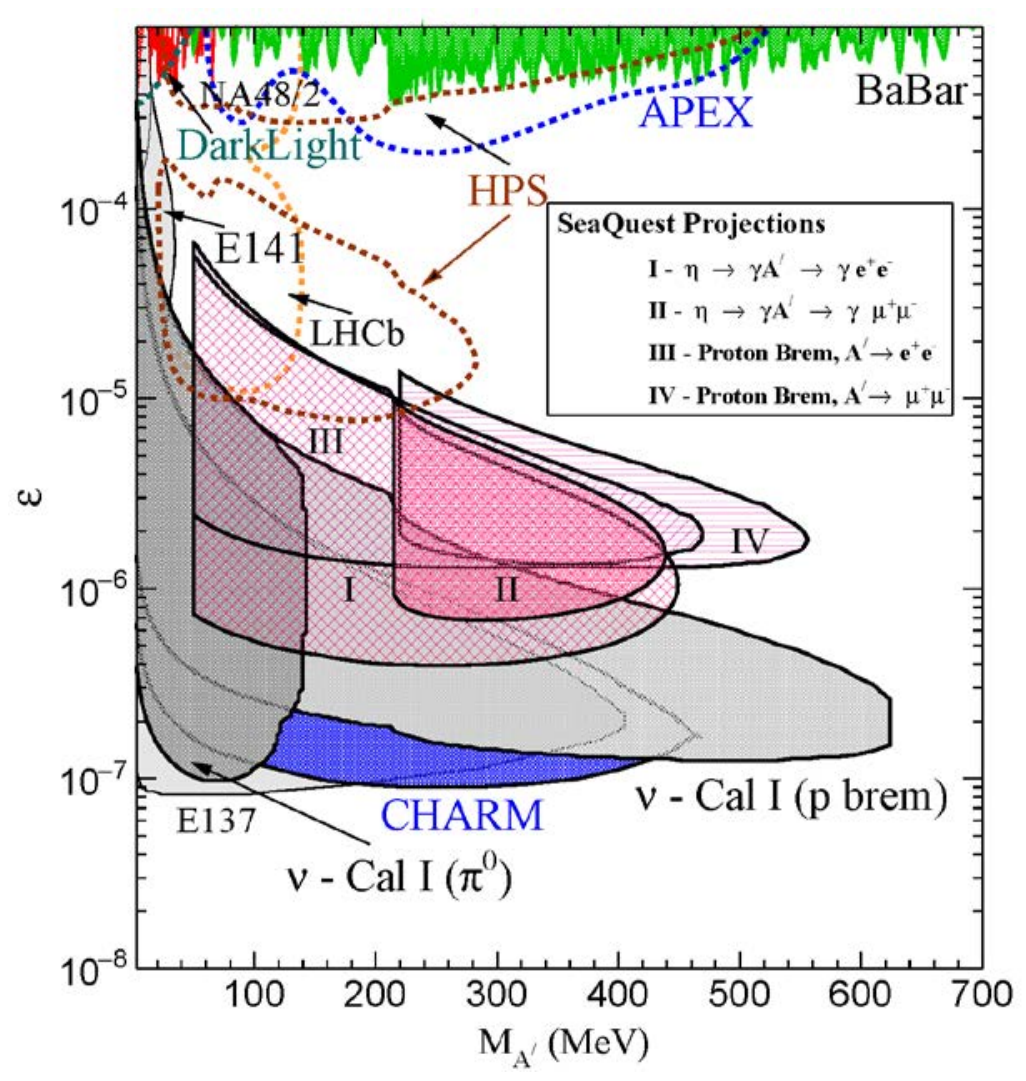

SeaQuest experimental parameters:

$\Rightarrow \mathrm{E}_{0}=5-110 \mathrm{GeV}$ for Proton Bremsstrahlung

$\Rightarrow \mathrm{N}_{\mathrm{eff}}=2$

$\Rightarrow \mathrm{I}_{0}=0.17 \mathrm{~m}-5.95 \mathrm{~m}$ 


\section{Polarized Proton Beams}

and Searches for Dark Forces

Searches for a dark photon also limit other possibilities

Parity violation studies could prove key

$$
\mathcal{L}_{\text {darkZ }}=-\left(\varepsilon e J_{\mathrm{em}}^{\mu}+\varepsilon_{Z} \frac{g}{2 \cos \theta_{W}} J_{\mathrm{NC}}^{\mu}\right) Z_{d \mu}
$$

[Davoudiasl, Lee, Marciano, 20I4]

If the $A^{\prime}$ is a dark $Z$, then ...

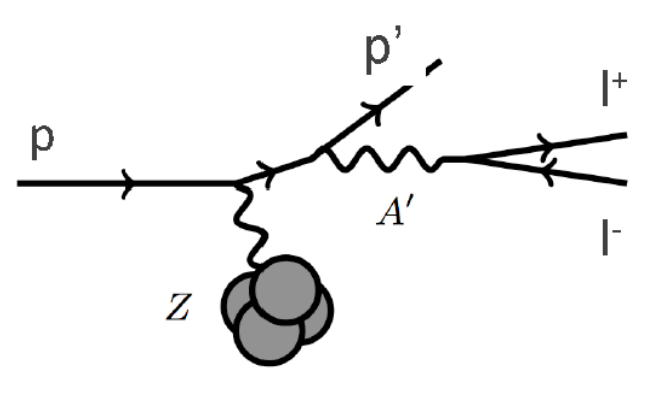

The dilepton yield can change with proton polarization: the asymmetry can be $\mathrm{O}(\mathrm{I})$ ! 


\section{Conclusions}

- There is an exciting Drell-Yan program with polarized/unpolarized beams and targets underway

$\longrightarrow$ although experimentally more challenging, it has some clear advantages over SIDIS

- Different labs offer complementary probes and processes to study hadronic landscape

$\rightarrow$ focus on strength of each lab to (minimize cost and) optimize physics output

- Future opportunities look very promising

$\rightarrow$ support from hadronic community (was and remains) vital to move forward

$\longrightarrow$ opportunities to join the Fermilab program

- We have finally seen first results from COMPASS on the sign-change

$\longrightarrow$ statistics still poor; but expect more in 2018

- Now entering an era where we will have first measurement of a sea quark Sivers function (answer some of the questions):

$\rightarrow$ How much do the quarks and gluons contribute to the nucleon spin?

$\longrightarrow$ In particular, what is the role of the sea quarks?

$\rightarrow$ Is there significant orbital angular momentum?

$\rightarrow$ Does TMD formalism work? Does Sivers function change sign (but keep shape and size)? 


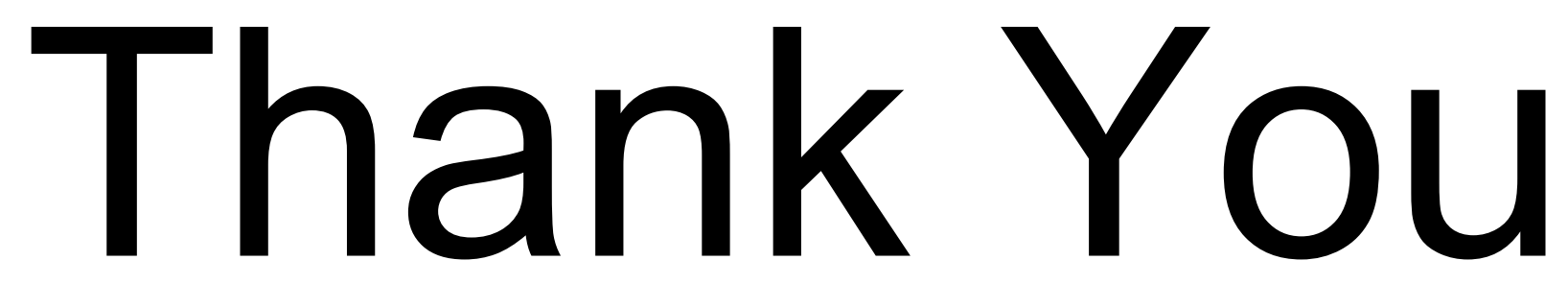

